\title{
Electromagnetic Structure of the Trinucleons
}

\author{
L.E. Marcucci \\ Department of Physics, Old Dominion University, Norfolk, Virginia 23529 \\ D.O. Riska \\ Department of Physics, University of Helsinki, SF-00014 Helsinki, Finland \\ R. Schiavilla \\ Jefferson Lab, Newport News, Virginia 23606 \\ and \\ Department of Physics, Old Dominion University, Norfolk, Virginia 23529
}

(July 18, 2021)

\begin{abstract}
The electromagnetic form factors of the trinucleons ${ }^{3} \mathrm{H}$ and ${ }^{3} \mathrm{He}$ are calculated with wave functions obtained with the Argonne $v_{18}$ two-nucleon and Urbana IX three-nucleon interactions. Full account is taken of the two-body currents required by current conservation with the $v_{18}$ interaction as well as those associated with $N \Delta$ transition currents and the currents of $\Delta$ resonance components in the wave functions. Explicit three-nucleon current operators associated with the two-pion exchange three-nucleon interaction arising from irreducible $S$-wave pion-nucleon scattering is constructed and shown to have very little effect on the calculated magnetic form factors. The calculated magnetic form factor of ${ }^{3} \mathrm{H}$, and charge form factors of both ${ }^{3} \mathrm{H}$ and ${ }^{3} \mathrm{He}$ are in satisfactory agreement with the experimental data. However, the position of the zero in the magnetic form factor of ${ }^{3} \mathrm{He}$ is slightly underpredicted.
\end{abstract}

21.45.+v,25.30.Bf,27.10.+h 


\section{INTRODUCTION}

The electromagnetic form factors of the few-body nuclei, along with the deuteron structure functions and cross section for threshold electrodisintegration at backward angles, are the observables of choice for testing the quality of models for the nuclear interaction and the associated current operator, including its exchange current components. Such testing has become possible by the development of practical computational methods for numerical calculation of the wave functions of the few nucleon systems, which correspond to realistic phenomenological interaction models [1]. Employing such wave functions along with the the two-nucleon exchange current operators, which are required by the continuity equation and/or consistency with the interaction models, e.g., by Poincaré invariance, it has become possible to predict the experimental electron scattering observables of the few nucleon systems up to momentum transfers of about $2 \mathrm{GeV} / \mathrm{c}$ in an at least qualitatively satisfactory way.

Among the remaining open issues are the need for quantitative understanding of the form factors of the trinucleons in the region around and above their first zeros. While the long standing, unsettled issue of the behavior of the tensor polarization of the deuteron for momentum transfers above $3 \mathrm{fm}^{-1}$ appears close to settlement by high quality experimental work at the Thomas Jefferson National Accelerator Facility, there remains a need for potential model development in the case of the trinucleon systems. This is partly because of the remaining problem in quantitative understanding of the trinucleon form factors at high momentum transfer and partly because of the resilient open issues concerning the form of the three-nucleon interaction, which appears to be required for the understanding of the binding energies of the light-nuclei $(A \leq 7)$ ground states [2].

Here this question is investigated in several different ways. First, a numerically extensive calculation of the electromagnetic form factors of the trinucleons is presented with high precision variational wave functions, which correspond to the Argonne $v_{18}$ two-nucleon [3] and the Urbana IX three-nucleon [4] interactions. In this calculation the two-nucleon exchange current operators are constructed by the same method as used in the earlier calculations in Refs. [5.6], that employed the Argonne $v_{14}$ interaction [7]. Second, the irreducible threenucleon exchange current operator, which corresponds to the best understood part of the two-pion exchange three-nucleon interaction associated with $S$-wave pion nucleon scattering on the intermediate nucleon is constructed, and its matrix elements for the trinucleon bound states are shown to be very small for momentum transfer values below $1 \mathrm{GeV} / \mathrm{c}$. Third, a systematic treatment of $\Delta$-isobar configurations in the trinucleon ground states is made, and their effect on the trinucleon form factors are calculated with inclusion of all the associated and required exchange current operators.

The calculated magnetic form factor of ${ }^{3} \mathrm{H}$ is in fairly good agreement with the present experimental data once the exchange current contributions are included. That of ${ }^{3} \mathrm{He}$ agrees less well with the corresponding data at high values of momentum transfer, which is a consequence of the underpredicted position of the first zero in the form factor (it falls at $3.75 \mathrm{fm}^{-1}$, which is below the experimental range $4.2-4.4 \mathrm{fm}^{-1}$ ). This result is independent of the presence or absence of the $\Delta$-isobar configurations in the wave function model. The problem is likely to have its origin in a somewhat too weak overall strength of the model for the isovector part of the exchange current operator at large momentum transfer. As 
this is constructed so as to be consistent with the $v_{18}$ two-nucleon interaction, the ultimate origin of the problem with the high momentum behavior of the calculated isovector magnetic form factor of the trinucleons may reside with the potential model if not with some purely transverse exchange current mechanism that has not been considered. The effect of the irreducible three-nucleon exchange current operator on this form factor is very small. The finding that the $\Delta$-isobar configurations have a very small effect on the trinucleon form factors conforms to earlier results obtained with other potential models [8].

The calculated charge form factor of ${ }^{3} \mathrm{He}$ agrees very well with the experimental values over the measured range of momentum transfer, with an exception for its highest end. In the case of ${ }^{3} \mathrm{H}$ quantitative agreement with the experimental form factor is achieved only up to the position of the secondary maximum, above which region the calculated values are too large by factors 2-3. The exchange current contributions are essential for agreement with the experimental charge form factors.

This paper is divided into four main sections. Section II contains a description of the calculation of the electromagnetic form factors of the trinucleons using a purely nucleonic variational wave function constructed for the $v_{18}$ model augmented by the Urbana IX threenucleon interaction. The details of the hyperspherical variational model wave function are given in Subsection II-A. Subsection II-B contains the description of the model for the electromagnetic current operator including the exchange current operators. In Subsection II-C the irreducible three-nucleon exchange current operator, which corresponds to the main nonresonant two-pion exchange three-nucleon interaction, is derived. Finally Subsection II-D contains the form factor results obtained with this restricted model. In Section III the description of the extended model wave function and current operators that include the $\Delta$-isobar configurations in the wave function is given. The model for the $N \Delta$ transition potential is described in Subsection III-A and the corresponding current operators are described in Subsection III-B. The calculation of the form factors in the extended model is outlined in Subsections III-C and III-D. Finally Section IV contains a concluding discussion.

\section{TRINUCLEON FORM FACTORS WITH NUCLEONIC WAVE FUNCTIONS}

In this section the calculation of the elastic form factors of the $A=3$ nuclei with wave functions for a realistic Hamiltonian model formed of the Argonne $v_{18}$ (AV18) two-nucleon [3] and Urbana IX (UIX) three-nucleon [4] interactions is described. The calculation employs charge and current operators that besides the standard single nucleon components also contain two-nucleon components, the leading terms of which are constructed consistently with the AV18 model. The three-nucleon exchange current operator, which corresponds to the two-pion exchange three-nucleon interaction associated with isospin odd $S$-wave pion

rescattering is also derived and is shown to have only a minor effect on the trinucleon form factors.

\section{A. The AV18/UIX Hamiltonian and trinucleon wave functions}

The AV18 model [3] is a recent high-quality nucleon-nucleon interaction containing explicit charge-symmetry-breaking (CSB) and charge-independence-breaking (CIB) terms, as 
well as a complete treatment of the electromagnetic interaction up to order $\alpha^{2}, \alpha$ being the fine structure constant. It is constructed to fit the Nijmegen $p p$ and $n p$ scattering database, low-energy $n n$ scattering parameters, and the deuteron binding energy with a $\chi^{2}$ per datum close to 1.

The UIX three-nucleon interaction [4] consists of a long-range term due to excitation of an intermediate $\Delta$-isobar via two-pion exchange and a short-range repulsive phenomenological term, which simulates the dispersive effects which arise upon integrating out $\Delta$-degrees of freedom. The strength of this repulsive term is determined by fitting the triton binding energy in "exact" Green's Function Monte Carlo (GFMC) calculations [2] and the equilibrium density of nuclear matter in variational calculations based on operator-chain summation techniques [9].

Recent GFMC calculations based on the AV18/UIX Hamiltonian model have been shown to provide a good description of the low-energy spectra and charge radii of nuclei with $A \leq 7$ [2]. In particular, the calculated binding energies of ${ }^{3} \mathrm{H}$ and ${ }^{3} \mathrm{He}$ are within a few $\mathrm{keV}$ of the experimental values (see Table $\mathbb{Q}$ ).

In the present work we use trinucleon wave functions obtained by Kievsky et al. [10,11, 12] with the pair-correlated hyperspherical harmonics $(\mathrm{PHH})$ method. Although variational this method has been refined in the last few years so much that it yields results with an accuracy comparable to that achieved in recent Faddeev and GFMC calculations, as may be seen in Table [. The PHH method is briefly reviewed here, for completeness; however, a more thorough discussion of it as well as its extensions to describe both the $A=3$ low energy continuum and $A=4$ ground state can be found in Refs. [11, 12, 13].

The wave function $\Psi$ of a three-nucleon system with total angular momentum $J J_{z}$ and total isospin $T T_{z}$ can be decomposed as

$$
\Psi=\sum_{i=1}^{3} \psi\left(\mathbf{x}_{i}, \mathbf{y}_{i}\right),
$$

where the amplitude $\psi\left(\mathbf{x}_{i}, \mathbf{y}_{i}\right)$ is a function of the Jacobi coordinates $\mathbf{x}_{i}=\mathbf{r}_{j}-\mathbf{r}_{k}$ and $\mathbf{y}_{i}=\left(\mathbf{r}_{j}+\mathbf{r}_{k}-2 \mathbf{r}_{i}\right) / \sqrt{3}, i, j, k$ being a cyclic permutation of $1,2,3$. To ensure the overall antisymmetry of $\Psi$, the amplitude $\psi\left(\mathbf{x}_{i}, \mathbf{y}_{i}\right)$ is antisymmetric with respect to exchange of nucleons $j$ and $k$. In the PHH method, it is expressed as [11,12

$$
\begin{gathered}
\psi\left(\mathbf{x}_{i}, \mathbf{y}_{i}\right)=\sum_{\alpha} f_{\alpha}\left(x_{i}\right) \Phi_{\alpha}\left(x_{i}, y_{i}\right) \mathcal{Y}_{\alpha}(j, k ; i) \\
\mathcal{Y}_{\alpha}(j, k ; i)=\left\{\left[Y_{\ell_{\alpha}}\left(\widehat{\mathbf{x}}_{i}\right) \otimes Y_{L_{\alpha}}\left(\widehat{\mathbf{y}}_{i}\right)\right]_{\Lambda_{\alpha}} \otimes\left[S_{\alpha}^{j k} \otimes s^{i}\right]_{S_{\alpha}}\right\}_{J J_{z}}\left[T_{\alpha}^{j k} \otimes t^{i}\right]_{T T_{z}},
\end{gathered}
$$

where each channel $\alpha$ is specified by the orbital angular momenta $\ell_{\alpha}, L_{\alpha}$ and $\Lambda_{\alpha}$, the spin (isospin) $S_{\alpha}^{j k}\left(T_{\alpha}^{j k}\right)$ of pair $j k$ and the total spin $S_{\alpha}$. Orbital and spin angular momenta are coupled, in the LS-scheme, to give total angular momenta $J J_{z}$. The (channel-dependent) correlation functions $f_{\alpha}\left(x_{i}\right)$ are obtained from solutions of two-body Schrödinger-like equations in channel $j_{\beta} l_{\beta} S_{\beta}^{j k} T_{\beta}^{j k}$ [10,11], and take into account the strong state-dependent correlations induced by the nucleon-nucleon interaction. They improve the behavior of the wave function at small interparticle distances. Were it not for their presence, the decomposition in Eq. (2.2) would be identical to that in the Faddeev scheme [14]. 
Next, the hyperspherical coordinates $\rho$ and $\phi_{i}$, defined as

$$
\rho=\sqrt{x_{i}^{2}+y_{i}^{2}}, \quad \cos \phi_{i}=x_{i} / \rho
$$

are introduced, and the dependence of $\Phi_{\alpha}\left(x_{i}, y_{i}\right)$ on $\rho$ and $\phi_{i}$ is made explicit by writing

$$
\begin{gathered}
\Phi_{\alpha}\left(x_{i}, y_{i}\right)=\sum_{n=0}^{M_{\alpha}} u_{n}^{\alpha}(\rho) Z_{n}^{\alpha}\left(\phi_{i}\right), \\
Z_{n}^{\alpha}\left(\phi_{i}\right)=N_{n}^{\ell_{\alpha}, L_{\alpha}}\left(\cos \phi_{i}\right)^{\ell_{\alpha}}\left(\sin \phi_{i}\right)^{L_{\alpha}} P_{n}^{\ell_{\alpha}+\frac{1}{2}, L_{\alpha}+\frac{1}{2}}\left(\cos 2 \phi_{i}\right),
\end{gathered}
$$

where $N_{n}^{\ell_{\alpha}, L_{\alpha}}$ are normalization factors, $P_{n}^{\alpha, \beta}$ are Jacobi polinomials and $n$ is a non-negative integer, $n=0, \cdots, M_{\alpha}$, where $M_{\alpha}$ is the selected number of basis functions in channel $\alpha$. The complete wave function is then written as

$$
\Psi=\sum_{i j k \text { cyclic }} \sum_{\alpha} f_{\alpha}\left(x_{i}\right) \mathcal{Y}_{\alpha}(j, k ; i) \sum_{n=0}^{M_{\alpha}} u_{n}^{\alpha}(\rho) Z_{n}^{\alpha}\left(\phi_{i}\right) .
$$

The Rayleigh-Ritz variational principle,

$$
\left\langle\delta_{u} \Psi|H-E| \Psi\right\rangle=0,
$$

is used to determine the hyper-radial functions $u_{n}^{\alpha}(\rho)$ in Eq. (2.7). Carrying out the variation $\delta_{u} \Psi$ with respect to the functions $u_{n}^{\alpha}(\rho)$, the following equation is easily derived:

$$
\left.\sum_{i j k \text { cyclic }}\left\langle f_{\alpha}\left(x_{i}\right) \mathcal{Y}_{\alpha}(j, k ; i) Z_{n}^{\alpha}\left(\phi_{i}\right)|H-E| \Psi\right\rangle\right|_{\Omega}=0,
$$

where $\Omega$ denotes the angular variables $\phi_{i}, \hat{\mathbf{x}}_{i}$ and $\hat{\mathbf{y}}_{i}$. Performing the integration over $\Omega$ and spin-isospin sums (as implicitly understood by the notation $\langle\cdots\rangle \mid \Omega$ ) leads to a set of coupled second order differential equations for the $u_{n}^{\alpha}(\rho)$, which is then solved by standard numerical techniques [10,11].

The binding energy of the $A=3$ nuclei obtained with the PHH method from the AV18/UIX Hamiltonian are listed in Table [ [12]. Also listed in Table $\mathrm{I}$ are results calculated with converged $r$-space [15] and $p$-space [16] Faddeev wave functions for an older model of the two-nucleon interaction, the Argonne $v_{14}$ (AV14) [7]. The binding energies obtained with the various methods are in excellent agreement with each other, typically within $10 \mathrm{keV}$ or less.

\section{B. Nuclear charge and current operators}

A fairly complete decription of the model for the nuclear electromagnetic current has been most recently given in Ref. [1]. Here we only review its general structure. The nuclear charge and current operators are expanded into a sum of one- and two-body terms: 


$$
\begin{aligned}
& \rho(\mathbf{q})=\sum_{i} \rho_{i}^{(1)}(\mathbf{q})+\sum_{i<j} \rho_{i j}^{(2)}(\mathbf{q}) \\
& \mathbf{j}(\mathbf{q})=\sum_{i} \mathbf{j}_{i}^{(1)}(\mathbf{q})+\sum_{i<j} \mathbf{j}_{i j}^{(2)}(\mathbf{q})
\end{aligned}
$$

where $\mathbf{q}$ is the momentum transfer. The one-body operators $\rho^{(1)}$ and $\mathbf{j}^{(1)}$ are given by

$$
\begin{aligned}
\rho_{i}^{(1)}(\mathbf{q})= & \frac{1}{\sqrt{1+q_{\mu}^{2} / 4 m^{2}}} \frac{1}{2}\left[G_{E}^{S}\left(q_{\mu}^{2}\right)+G_{E}^{V}\left(q_{\mu}^{2}\right) \tau_{z, i}\right] e^{\mathrm{i} \mathbf{q} \cdot \mathbf{r}_{i}} \\
& -\frac{\mathrm{i}}{8 m^{2}}\left[2 G_{M}^{S}\left(q_{\mu}^{2}\right)-G_{E}^{S}\left(q_{\mu}^{2}\right)+\left[2 G_{M}^{V}\left(q_{\mu}^{2}\right)-G_{E}^{V}\left(q_{\mu}^{2}\right)\right] \tau_{z, i}\right] \mathbf{q} \cdot\left(\boldsymbol{\sigma}_{i} \times \mathbf{p}_{i}\right) e^{\mathrm{i} \mathbf{q} \cdot \mathbf{r}_{i}}, \\
\mathbf{j}_{i}(\mathbf{q})= & \frac{1}{4 m}\left[G_{E}^{S}\left(q_{\mu}^{2}\right)+G_{E}^{V}\left(q_{\mu}^{2}\right) \tau_{z, i}\right]\left\{\mathbf{p}_{i}, e^{\mathrm{i} \mathbf{q} \cdot \mathbf{r}_{i}}\right\} \\
& -\frac{\mathrm{i}}{4 \mathrm{~m}}\left[G_{M}^{S}\left(q_{\mu}^{2}\right)+G_{M}^{V}\left(q_{\mu}^{2}\right) \tau_{z, i}\right] \mathbf{q} \times \boldsymbol{\sigma}_{i} e^{\mathrm{i} \mathbf{q} \cdot \mathbf{r}_{i}}
\end{aligned}
$$

up to terms proportional to $1 / m^{2}, m$ being the nucleon mass. Equation (2.12) includes the leading relativistic corrections to single-nucleon charge operator, namely the Darwin-Foldy and spin orbit terms. Here the $G_{E / M}^{S / V}\left(q_{\mu}^{2}\right)$ are the electric/magnetic $(E / M)$ isoscalar/isovector $(S / V)$ form factors of the nucleon, taken as function of the four-momentum transfer

$$
q_{\mu}^{2}=\mathbf{q}^{2}-\omega^{2}>0
$$

where the energy transfer $\omega=\sqrt{\mathbf{q}^{2}+m_{T}^{2}}-m_{T}$ for elastic scattering on a target of mass $m_{T}$ initially at rest in the lab. These form factors are normalized as

$$
\begin{aligned}
G_{E}^{S}(0) & =G_{E}^{V}(0)=1, \\
G_{M}^{S}(0) & =0.880 \mu_{N}, \\
G_{M}^{V}(0) & =4.706 \mu_{N},
\end{aligned}
$$

$\mu_{N}$ being the nuclear magneton, and their $q_{\mu}$-dependence is constrained by analyzing electron-proton and electron-deuteron scattering data. While the proton electric and magnetic form factors are experimentally fairly well known over a wide range of momentum transfers, there is significant uncertainty in the neutron form factors, particularly the electric one, which are obtained from model-dependent analyses of ed data. Until this uncertainty in the detailed behaviour of the electromagnetic form factors of the nucleon is narrowed, quantitative predictions of electro-nuclear observables at high momentum transfers will remain rather tentative. We will re-examine this issue in Sec. II-D-1 below.

\section{The two-body current operator}

The two-body current operator can be separated into a model-independent (MI) term determined from the interaction (in the present case, the charge-independent part of the AV18 model) following a prescription originally proposed in Ref. [17], and a model-dependent (MD) one, associated with the $\rho \pi \gamma$ and $\omega \pi \gamma$ electromagnetic couplings. Explicit expressions for all these currents have been most recently given in Ref. [18]. 
The $\rho \pi \gamma$ and $\omega \pi \gamma \mathrm{MD}$ currents are purely transverse and therefore unconstrained by the nucleon-nucleon interaction. The values of the transition form factors $G_{\rho \pi \gamma}\left(q_{\mu}^{2}\right)$ and $G_{\omega \pi \gamma}\left(q_{\mu}^{2}\right)$ at the photon point are known to be $G_{\rho \pi \gamma}(0)=g_{\rho \pi \gamma}=0.56$, Ref. [19], and $G_{\omega \pi \gamma}(0)=g_{\omega \pi \gamma}=0.68$, Ref. [20], from the measured widths of the $\rho \rightarrow \pi \gamma$ and $\omega \rightarrow \pi \gamma$ decays, while their $q_{\mu}$-dependence is modeled using vector-meson dominance. Monopole form factors at the pion and vector-meson strong interaction vertices are introduced to take into account the composite nature of nucleons and mesons. The cutoff parameters $\Lambda_{\pi}, \Lambda_{\rho}$ and $\Lambda_{\omega}$ in these form factors are not known. Here we use the values $\Lambda_{\pi}=0.75 \mathrm{GeV}$ and $\Lambda_{\rho}=\Lambda_{\omega}=1.25 \mathrm{GeV}$ obtained from studies of the B-structure function of the deuteron [21].

The leading MI two-body currents, denoted as pseudoscalar (PS) or $\pi$-like and vector (V) or $\rho$-like, are the isovector ones associated with the isospin-dependent central, spin-spin, and tensor components of the interaction. Their derivation has been given in a number of references [1,22], and will not be repeated here. We only note that: i) the PS and V twobody currents have no free parameters and, by construction, satisfy the continuity equation with the given realistic interaction (here the charge-independent part of AV18 model); ii) the continuity equation requires the same form factor be used to describe the electromagnetic structure of the hadrons in the longitudinal part of the current operator and in the charge operator, while it places no restrictions on the electromagnetic form factors which may be used in the transverse parts of the current. Ignoring this ambiguity, the form factor $G_{E}^{V}\left(q_{\mu}^{2}\right)$ is used in the PS and V currents operators, in line with the "minimal" requirements of current conservation.

There are additional two-body currents associated with the momentum dependence of the interaction, but their construction is less straightforward. A procedure similar to that used to derive the PS and V currents has been generalized to the case of the currents from spin-orbit components of the interaction [23]. It consists, in essence, of attributing these to exchanges of $\sigma$-like and $\omega$-like mesons for the isospin-independent terms, and to $\rho$-like mesons for the isospin-dependent ones. The explicit form of the resulting currents, denoted as SO, can be found in Refs. [18,23]. The two-body currents from the quadratic momentum dependence of the interaction are obtained by minimal substitution $\mathbf{p}_{i} \rightarrow \mathbf{p}_{i}-$ $\frac{1}{2}\left[G_{E}^{S}\left(q_{\mu}^{2}\right)+G_{E}^{V}\left(q_{\mu}^{2}\right) \tau_{z, i}\right] \mathbf{A}\left(\mathbf{r}_{i}\right), \mathbf{A}\left(\mathbf{r}_{i}\right)$ being the vector potential, into the corresponding components. In the case of the AV18 model, the $p^{2}$-dependence is via $\mathbf{L}^{2}$ and $\left(\mathbf{L} \cdot \boldsymbol{\sigma}_{1} \mathbf{L} \cdot \boldsymbol{\sigma}_{2}+\right.$ h.c.) terms, and the associated currents are denoted respectively as LL and SO2 [18,5].

We note that the SO, LL and SO2 currents are fairly short-ranged, and have both isoscalar and isovector terms. Their contribution to isovector observables is found to be numerically much smaller than that due to the leading PS ( $\pi$-like) current. However, these currents give non-negligible corrections to isoscalar observables, such as the deuteron magnetic moment and B-structure function [24]. Finally it is worth enphasizing that, while the construction in Ref. [22] is not unique, it has nevertheless been shown to provide, at low and moderate values of momentum transfer, satisfactory description of most observables where the isovector two-body currents play a large (if not dominant) role, such as the deuteron threshold electrodisintegration [24], the neutron and proton radiative captures on protons and deuterons at low energies [18,24, and the magnetic moments and form factors of the trinucleons (as shown below). 


\section{Two-body charge operators}

While the MI two-body currents are linked to the form of nucleon-nucleon interaction via the continuity equation, the most important two-body charge operators are model dependent and may be viewed as relativistic corrections. They fall into two classes. The first class includes those effective operators that represent non-nucleonic degrees of freedom, such as nucleon-antinucleon pairs or nucleon-resonances, and which arise when these degrees of freedom are eliminated from the state vector. To the second class belong those dynamical exchange charge effects that would appear even in a description explicitly including nonnucleonic excitations in the state vector, such as the $\rho \pi \gamma$ and $\omega \pi \gamma$ transition couplings. The proper forms of the former operators depend on the method of eliminating the non-nucleonic degrees of freedom [25,26,27]. There are nevertheless rather clear indications for the relevance of two-body charge operators from the failure of calculations based on the one-body operator in Eq. (2.12) in predicting the charge form factors of the three- and four-nucleon systems [6], and deuteron A-structure function and tensor polarization observable 24, 28.

The two-body model used in the present work consists of the $\pi-, \rho$ - and $\omega$-meson exchange charge operators, as well as of the $\rho \pi \gamma$ and $\omega \pi \gamma$ charge transition couplings. The former are derived by considering the low-energy limit of the relativistic Born diagrams associated with the virtual meson photoproduction amplitude. The $\rho \pi \gamma$ and $\omega \pi \gamma$ operators are the leading corrections obtained in a non-relativistic reduction of the corresponding Feynman diagrams with transition couplings, for example $\left\langle\pi^{a}(k)\left|j_{\mu}(0)\right| \rho^{b}(p, \epsilon)\right\rangle=$

$-\left[G_{\rho \pi \gamma}\left(q_{\mu}^{2}\right) / m_{\rho}\right] \delta_{a b} \epsilon_{\mu \nu \sigma \tau} p^{\nu} k^{\sigma} \epsilon^{\tau}, \epsilon$ being the polarization vector of the $\rho$-meson. Coupling constants and cutoff parameters are given in the previous subsection. Explicit expression for all these operators can be found in Ref. [6]. Here we only note that: i) the $\pi$ - and $\rho$ meson exchange charge operators, the former of which gives by far the dominant contribution, are constructed using the PS ( $\pi$-like) and V ( $\rho$-like) components projected out of the isospin-dependent spin-spin and tensor terms of the interaction [6], thus reducing their model dependence. The resulting two-body operators are denoted as PS and V, and are here obtained from the charge-independent part of the AV18. ii) In the pion (as well as vector meson) charge operators there are additional contributions due to the energy dependence of the pion propagator and direct coupling of the photon to the exchanged pion ( $\rho$-meson). However, these operators give rise to non-local isovector contributions which are expected to provide only small corrections to the leading local terms. For example these operators would only contribute to the isovector combination of the ${ }^{3} \mathrm{He}$ and ${ }^{3} \mathrm{H}$ charge form factors, which is anyway a factor of three smaller than the isoscalar. Thus they are neglected in the present work.

\section{The three-body exchange current associated with $S$-wave pion rescattering}

The isospin odd "large" component of the $S$-wave pion-nucleon $(\pi N)$ scattering amplitude at low energy and momentum transfer may be described by the effective interaction [29]:

$$
\mathcal{L}_{\pi \pi N N}=-\frac{1}{4 f_{\pi}^{2}} \bar{\psi} \gamma^{\mu} \boldsymbol{\tau} \cdot \psi \boldsymbol{\phi} \times \partial_{\mu} \boldsymbol{\phi} .
$$


Here $\boldsymbol{\phi}$ is the isovector pion field and $f_{\pi}$ the pion decay constant $(\simeq 93 \mathrm{MeV})$. This effective Lagrangian implies the "Weinberg-Tomozawa" relation for the isospin odd combination of the $\pi N S$-wave scattering lengths $a_{1}, a_{3}$ :

$$
\lambda_{2}=\frac{1}{6}\left(1+\frac{m_{\pi}}{m}\right)\left(a_{1}-a_{3}\right)=\frac{1}{16 \pi}\left(\frac{m_{\pi}}{f_{\pi}}\right)^{2},
$$

which agrees well with the experimental scattering length values. Combined with the pseudovector $\pi N N$ interaction

$$
\mathcal{L}_{\pi N N}=-\frac{f_{\pi N N}}{m_{\pi}} \bar{\psi} \gamma^{5} \gamma^{\mu} \boldsymbol{\tau} \psi \cdot \partial_{\mu} \boldsymbol{\phi}
$$

where $f_{\pi N N} \simeq 1$, this interaction gives rise to the three-body interaction:

$$
\begin{aligned}
V_{S}= & -\frac{1}{4 m} \frac{1}{f_{\pi}^{2}}\left(\frac{f_{\pi N N}}{m_{\pi}}\right)^{2} \sum_{i j k \text { cyclic }} \boldsymbol{\tau}_{i} \cdot \boldsymbol{\tau}_{j} \times \boldsymbol{\tau}_{k} \frac{\boldsymbol{\sigma}_{i} \cdot \mathbf{k}_{i} \boldsymbol{\sigma}_{k} \cdot \mathbf{k}_{k}}{D_{i} D_{k}} \\
& \left\{\boldsymbol{\sigma}_{j} \cdot \mathbf{k}_{i} \times \mathbf{k}_{k}+\frac{\mathrm{i}}{2}\left[\mathbf{k}_{i} \cdot\left[\left(\mathbf{p}_{i}+\mathbf{p}_{i}^{\prime}\right)-\left(\mathbf{p}_{j}+\mathbf{p}_{j}^{\prime}\right)\right]-\mathbf{k}_{k} \cdot\left[\left(\mathbf{p}_{k}+\mathbf{p}_{k}^{\prime}\right)-\left(\mathbf{p}_{j}+\mathbf{p}_{j}^{\prime}\right)\right]\right]\right\} .
\end{aligned}
$$

The momentum vectors are defined so that $\mathbf{k}_{i}$ denotes the fractional momentum transfer to nucleon $i$. The denominator factors $D_{i}$ are defined as

$$
D_{i}=\mathbf{k}_{i}^{2}+m_{\pi}^{2}
$$

The derivative couplings in the Lagrangians (2.16) and (2.19) lead to electromagnetic contact terms. These may be constructed by minimal substitution, and are found to have the expressions

$$
\begin{gathered}
\mathcal{L}_{\pi \pi \gamma N N}=-\frac{1}{4 f_{\pi}^{2}} \bar{\psi} \gamma^{\mu} A_{\mu}\left[\phi_{z}(\boldsymbol{\tau} \cdot \boldsymbol{\phi})-\tau_{z} \boldsymbol{\phi}^{2}\right] \psi, \\
\mathcal{L}_{\pi \gamma N N}=-\frac{f_{\pi N N}}{m_{\pi}} \bar{\psi} \gamma^{5} \gamma^{\mu} A_{\mu}(\boldsymbol{\tau} \times \boldsymbol{\phi})_{z} \psi,
\end{gathered}
$$

respectively. When complemented with the electromagnetic coupling of the pion,

$$
\mathcal{L}_{\pi \pi \gamma}=-A_{\mu}\left(\phi \times \partial^{\mu} \phi\right)_{z}
$$

these contact terms give rise to the following set of three-nucleon exchange current operators: (a) a contact current at the $S$-wave rescattering vertex, (b) two contact currents at the two accompanying pseudovector $\pi N N$ vertices and (c) two pion current terms. The explicit expressions for these are in the corresponding order:

$$
\begin{aligned}
\mathbf{j}_{i j k}^{a}(\mathbf{q})= & \frac{\mathrm{i}}{8 m} \frac{1}{f_{\pi}^{2}}\left(\frac{f_{\pi N N}}{m_{\pi}}\right)^{2}\left[\boldsymbol{\tau}_{k} \times\left(\boldsymbol{\tau}_{j} \times \boldsymbol{\tau}_{i}\right)+\boldsymbol{\tau}_{i} \times\left(\boldsymbol{\tau}_{j} \times \boldsymbol{\tau}_{k}\right)\right]_{z} \\
& \frac{\left(\boldsymbol{\sigma}_{i} \cdot \mathbf{k}_{i}\right)\left(\boldsymbol{\sigma}_{k} \cdot \mathbf{k}_{k}\right)}{D_{i} D_{k}}\left[\boldsymbol{\sigma}_{j} \times\left(\mathbf{q}-\mathbf{k}_{i}-\mathbf{k}_{k}\right)-\mathrm{i}\left(\mathbf{p}_{j}+\mathbf{p}_{j}^{\prime}\right)\right],
\end{aligned}
$$




$$
\begin{aligned}
\mathbf{j}_{i j k}^{b}(\mathbf{q})= & \frac{\mathrm{i}}{4 m} \frac{1}{f_{\pi}^{2}}\left(\frac{f_{\pi N N}}{m_{\pi}}\right)^{2}\left[\boldsymbol{\tau}_{i} \times\left(\boldsymbol{\tau}_{j} \times \boldsymbol{\tau}_{k}\right)\right]_{z} \frac{\boldsymbol{\sigma}_{i}\left(\boldsymbol{\sigma}_{k} \cdot \mathbf{k}_{k}\right)}{D_{k} D_{i}^{\prime}}\left\{\left[\boldsymbol{\sigma}_{j} \cdot\left(\mathbf{k}_{i}-\mathbf{q}\right) \times \mathbf{k}_{k}\right]\right. \\
& +\frac{\mathrm{i}}{2}\left[\mathbf{k}_{i} \cdot\left[\left(\mathbf{p}_{i}+\mathbf{p}_{i}^{\prime}\right)-\left(\mathbf{p}_{j}+\mathbf{p}_{j}^{\prime}\right)\right]-\mathbf{k}_{k} \cdot\left[\left(\mathbf{p}_{k}+\mathbf{p}_{k}^{\prime}\right)-\left(\mathbf{p}_{j}+\mathbf{p}_{j}^{\prime}\right)\right]\right. \\
& \left.\left.-2 m \omega+\mathbf{q} \cdot\left(\mathbf{p}_{j}+\mathbf{p}_{j}^{\prime}\right)\right]\right\}+(i \rightleftharpoons k), \\
\mathbf{j}_{i j k}^{c}(\mathbf{q})= & -\frac{\mathrm{i}}{4 m} \frac{1}{f_{\pi}^{2}}\left(\frac{f_{\pi N N}}{m_{\pi}}\right)^{2}\left[\boldsymbol{\tau}_{i} \times\left(\boldsymbol{\tau}_{j} \times \boldsymbol{\tau}_{k}\right)\right]_{z} \frac{\left(\boldsymbol{\sigma}_{i} \cdot \mathbf{k}_{i}\right)\left(\boldsymbol{\sigma}_{k} \cdot \mathbf{k}_{k}\right)}{D_{i} D_{k}} \frac{2 \mathbf{k}_{i}-\mathbf{q}}{D_{i}^{\prime}} \\
& \left\{\left[\boldsymbol{\sigma}_{j} \cdot\left(\mathbf{k}_{i}-\mathbf{q}\right) \times \mathbf{k}_{k}\right]+\frac{\mathrm{i}}{2}\left[\mathbf{k}_{i} \cdot\left[\left(\mathbf{p}_{i}+\mathbf{p}_{i}^{\prime}\right)-\left(\mathbf{p}_{j}+\mathbf{p}_{j}^{\prime}\right)\right]\right.\right. \\
- & \left.\left.\mathbf{k}_{k} \cdot\left[\left(\mathbf{p}_{k}+\mathbf{p}_{k}^{\prime}\right)-\left(\mathbf{p}_{j}+\mathbf{p}_{j}^{\prime}\right)\right]-2 m \omega+\mathbf{q} \cdot\left(\mathbf{p}_{j}+\mathbf{p}_{j}^{\prime}\right)\right]\right\}+(i \rightleftharpoons k) .
\end{aligned}
$$

In these exchange current operators the fractions of the total momentum transfer $\mathbf{q}$ imparted to the three nucleons are denoted $\mathbf{k}_{i}$ respectively so that $\mathbf{q}=\mathbf{k}_{1}+\mathbf{k}_{2}+\mathbf{k}_{3}$. The denominator factors $D_{i}$ are defined in (2.20), while the denominator factors $D_{i}{ }^{\prime}$ are defined as

$$
D_{i}^{\prime}=\left(\mathbf{q}-\mathbf{k}_{i}\right)^{2}+m_{\pi}^{2} .
$$

The combined three-nucleon exchange current operator $\mathbf{j}^{a}+\mathbf{j}^{b}+\mathbf{j}^{c}$ satifies the continuity equation with the three-nucleon interaction $V_{S}$ (2.19), as may be verified by comparing the product $\mathbf{q} \cdot \mathbf{j}$ with the commutator of $V_{S}$ and the single nucleon charge operator. These two-pion exchange three-nucleon currents will be labelled as $\pi \pi_{S}$ below.

Note that the three-nucleon interaction (2.19) is not contained in the Urbana IX threenucleon interaction model [4], the main part of which takes into account exchanges that involve excitation of intermediate $\Delta$-isobar resonances, which are treated explicitly below. It should however be included in any complete three-nucleon interaction model, as it is implied by effective chiral Lagrangian models for the pion-nucleon system. It is included in three-nucleon interaction models that are based on pion exchange and rescattering described by current algebra or chiral Lagrangians (cf in the " $\mathrm{d}$ " term of the three-nucleon interaction in Ref. [30]).

\section{Elastic form factors of ${ }^{3} \mathrm{H}$ and ${ }^{3} \mathrm{He}$}

In this section we present results for the magnetic moments, charge and magnetic form factors of ${ }^{3} \mathrm{H}$ and ${ }^{3} \mathrm{He}$. The nuclear ground states are described by the $\mathrm{PHH}$ wave function obtained from the AV18/UIX Hamiltonian model.

A convenient espression to calculate the magnetic form factors of a $J=1 / 2$ nucleus, such as the $A=3$ systems under consideration here, is obtained by orienting the coordinate system

so that the spin-quantization axis (the $z$-axis) lies along the momentum transfer $\mathbf{q}$. It is then found that

$$
F_{M}(q)=\frac{2 m}{\mu} \frac{1}{q}\left\langle\Psi_{+}\left|j_{x}(q \hat{\mathbf{z}})\right| \Psi_{-}\right\rangle
$$


where $\mu$ is the nuclear magnetic moment in terms of $\mu_{N}$ (the nuclear magneton), $\Psi_{+/-}$are the normalized ground state wave functions with $J_{z}= \pm 1 / 2$, respectively, and $j_{x}(q \hat{\mathbf{z}})$ is the $x$-component of the current operator. Note that $F_{M}(0)=1$. The charge form factor is easily obtained from

$$
F_{C}(q)=\frac{1}{Z}\left\langle\Psi_{+}|\rho(q \hat{\mathbf{z}})| \Psi_{+}\right\rangle
$$

with $F_{C}(0)=1$.

The matrix elements (2.28) and (2.29) are evaluated with Monte Carlo methods. The wave function is written as a vector in the spin-isospin space of the three nucleons for any given spatial configuration $\mathbf{R} \equiv\left(\mathbf{r}_{1}, \mathbf{r}_{2}, \mathbf{r}_{3}\right)$. For the given $\mathbf{R}$, the state vectors $j_{x}(q \hat{\mathbf{z}})\left|\Psi_{-}\right\rangle$ and $\rho(q \hat{\mathbf{z}})\left|\Psi_{+}\right\rangle$are calculated by performing exactly the spin-isospin algebra with the techniques described in Refs. [5,6]. The spatial integrations are carried out by sampling the $\mathbf{R}$-configurations according to the Metropolis et al. algorithm [31]. Typically, 400,000 configurations are enough, in the form factor calculations reported here, to achieve a relative error of few $\%$ at low and moderate values of momentum transfer $q\left(q \leq 5 \mathrm{fm}^{-1}\right)$, increasing to $\sim 30 \%$ at the highest $q$-values.

\section{The magnetic form factors}

The current operator includes, in addition to the one-body current in Eq. (2.13), the MI two-body currents obtained from the charge-independent part of the AV18 interaction (denoted as PS or $\pi$-like, $\mathrm{V}$ or $\rho$-like, SO, LL and SO2), the MD $\rho \pi \gamma$ and $\omega \pi \gamma$ two-body currents, and finally the local terms of the three-body current associated with the $S$-wave two-pion exchange three-nucleon interaction (2.19) described in the previous section.

Because of destructive interference in the matrix element for the magnetic dipole transition between the $S$ - and $D$-state components of the wave function, the one-body predictions for the ${ }^{3} \mathrm{H}$ and ${ }^{3} \mathrm{He}$ magnetic form factors (MFF) have distinct minima at around $\sim 3.5 \mathrm{fm}^{-1}$ and $\sim 2.5 \mathrm{fm}^{-1}$, respectively, in disagreement with the experimental data [32,33,34,35,36,37,38,39,40,41], as shown in Figs. 11 and 2. The situation is closely related to that of the backward cross section for electrodisintegration of the deuteron, which is in fact dominated by two-body current contributions for values of momentum transfer above $\sim 2.5 \mathrm{fm}^{-1} 42$.

Inclusion of the contributions from the two- and $\pi \pi_{S}$ three-body currents shifts the zeros in the calculated MFF to higher $q$-values. While the experimental ${ }^{3} \mathrm{H} \mathrm{MFF}$ is in good agreement with theory over a wide range of momentum transfers, there is a significant discrepancy between the measured and calculated values of the ${ }^{3} \mathrm{He} \mathrm{MFF}$ in the region of diffraction minimum. This discrepancy persists even when different parametrizations of the nucleon electromagnetic form factors are used. This is evident from Figs. 1 and 2 where the total results obtained with the Gari-Krümpelmann (GK) parametrization [43] of the nucleon electromagnetic form factors are shown.

It is useful to define the quantities

$$
F_{M}^{S, V}(q)=\frac{1}{2}\left[\mu\left({ }^{3} \mathrm{He}\right) F_{M}\left(q ;{ }^{3} \mathrm{He}\right) \pm \mu\left({ }^{3} \mathrm{H}\right) F_{M}\left(q ;{ }^{3} \mathrm{H}\right)\right] .
$$


If the ${ }^{3} \mathrm{H}$ and ${ }^{3} \mathrm{He}$ ground states were pure $T=1 / 2$ states, then $F_{M}^{S}$ and $F_{M}^{V}$ linear combinations of the three-nucleon MFF would be only influenced by, respectively, the isoscalar $(S)$ and isovector $(V)$ parts of the current operator. However, small isospin admixtures with $T>1 / 2$, induced by electromagnetic, CSB and CIB terms present in the AV18 interaction, are included in the present wave functions. As a consequence, purely isoscalar (isovector) current operators give small, otherwise vanishing, contributions to the $F_{M}^{V}\left(F_{M}^{S}\right) \mathrm{MFF}$.

The contributions of the individual components of the two- and three-nucleon $\left(\pi \pi_{S}\right.$ term) currents to the $F_{M}^{S}$ and $F_{M}^{V}$ combinations are shown in Figs. 3 and 4 . In the diffraction region the PS ( $\pi$-like) isovector current gives the dominant contribution to $F_{M}^{V}$, while the contributions from remaining currents are significantly smaller, about one order of magnitude or more. The three-nucleon current $\left(\pi \pi_{S}\right)$ associated with the $S$-wave $\pi N$ coupling is found to give a very small correction.

Among the two-body contributions to $F_{M}^{S}$, the most important is that due to the currents from the spin-orbit interactions (SO), the remaining operators producing a very small correction. Note that the isovector PS and V currents contribute to $F_{M}^{S}$ because of the small isospin-symmetry breaking components present in the ${ }^{3} \mathrm{H}$ and ${ }^{3} \mathrm{He}$ wave functions induced by the AV18 model, as mentioned earlier.

Finally, the cumulative contributions to the $F_{M}^{S}$ and $F_{M}^{V}$ combinations are compared with the experimental data 41] in Figs. 5 and 6, respectively. The zero in the calculated $F_{M}^{V}$ is found to occur at lower $q$-value than experimentally observed. As shown in the next section, this discrepancy between theory and experiment remains unresolved even when $\Delta$-isobar degrees of freedom are included in both the nuclear wave functions and currents. We will return to this point in the conclusions. Predictions for the magnetic moments are given in Tables 【I and 【II, while those for the magnetic radii are listed in Table IV. These results are discussed in Sec. III-D.

\section{The charge form factors}

The charge operator includes, in addition to the one-body term of Eq. (2.12), the PS or $\pi$-like, $\mathrm{V}$ or $\rho$-like, $\omega, \rho \pi \gamma$ and $\omega \pi \gamma$ two-body operators, discussed previously. The calculated ${ }^{3} \mathrm{H}$ and ${ }^{3} \mathrm{He}$ charge form factors (CFF) are compared with the experimental data 32, 33, 34,35, 36, 37, 38, 39, 40,41 in Figs. 7 and 8. There is excellent agreement between theory and experiment, as is clear from these figures. The important role of the two-body contributions above $3 \mathrm{fm}^{-1}$ is also evident. The remarkable success of the present picture based on non-relativistic wave functions and a charge operator including the leading relativistic corrections should be stressed. It suggests, in particular, that the present model for the twobody charge operator is better than one a priori should expect. These operators, such as the PS charge operator, fall into the class of relativistic corrections. Thus, evaluating their matrix elements with non-relativistic wave functions represents only the first approximation to a systematic reduction. A consistent treatment of these relativistic effects would require, for example, inclusion of the boost corrections on the nuclear wave functions [25, 26, 44, Yet, the excellent agreement between the calculated and measured CFF suggests that these corrections may be neglegible in the $q$-range explored so far.

For completeness, we show in Figs. 9 and 10 the contributions from the individual components of the charge operator to the linear combinations 


$$
F_{C}^{S, V}(q)=\frac{1}{2}\left[2 F_{C}\left(q ;^{3} \mathrm{He}\right) \pm F_{C}\left(q ;^{3} \mathrm{H}\right)\right] .
$$

Note that again, because of isospin-symmetry breaking components present in the ${ }^{3} \mathrm{He}$ and ${ }^{3} \mathrm{H}$ wave functions, the purely isovector (isoscalar) $\omega \pi \gamma(\rho \pi \gamma)$ charge operator gives a small, otherwise vanishing, correction to the $F_{C}^{S}\left(F_{C}^{V}\right) \mathrm{CFF}$.

Finally, values for the charge radii of ${ }^{3} \mathrm{H}$ and ${ }^{3} \mathrm{He}$ are listed in Table $\nabla$. The results including the contributions associated with the two-body charge operators are found to be in good agreement with experimental data.

\section{BEYOND NUCLEONS ONLY}

The simplest picture views the nucleus as being made up of nucleons, and assumes that all other sub-nucleonic degrees of freedom may be eliminated in favor of effective many-body operators acting on the nucleons' coordinates. The validity of such a description is based on the success it has achieved in the quantitative prediction of many nuclear observables [1]. However, it is interesting to consider corrections to this picture by including the degrees of freedom associated with nuclear resonances as additional constituents of the nucleus. When treating phenomena which do not involve explicitely meson production, it is reasonable to expect that the lowest excitation of the nucleon, the $\Delta$-isobar, plays a leading role.

In such an approach, the $A=3$ nuclear wave function is written as

$$
\Psi_{N+\Delta}=\Psi(N N N)+\Psi^{(1)}(N N \Delta)+\Psi^{(2)}(N \Delta \Delta)+\Psi^{(3)}(\Delta \Delta \Delta),
$$

where $\Psi$ is that part of the total wave function consisting only of nucleons; the term $\Psi^{(1)}$ is the component in which a single nucleon has been converted into a $\Delta$-isobar, and so on. The nuclear two-body interaction is taken as

$$
v_{i j}=\sum_{B_{i}, B_{j}=N, \Delta} \sum_{B_{i}^{\prime}, B_{j}^{\prime}=N, \Delta} v_{i j}\left(B_{i} B_{j} \rightarrow B_{i}^{\prime} B_{j}^{\prime}\right),
$$

where transition interactions such as $v_{i j}(N N \rightarrow N \Delta), v_{i j}(N N \rightarrow \Delta \Delta)$, etc. are responsible for generating $\Delta$-isobar admixtures in the wave function. The long-range part of $v_{i j}$ is due to pion-exchange, while its short- and intermediate-range parts, influenced by more complex dynamics, is constrained by fitting $N N$ scattering data at lab energy $\leq 400 \mathrm{MeV}$ and deuteron properties [7].

Once the $N N, N \Delta$ and $\Delta \Delta$ interactions have been determined, the problem is reduced to solving the $N-\Delta$ coupled-channel Schrödinger equation. In principle, for the $A=3$ systems Faddeev and hyperspherical-harmonics techniques can be used (and, indeed, Faddeev methods have been used in the past [45,46]) to this end, although the large number of $N-\Delta$ channels involved makes the practical implementation of these methods difficult. A somewhat simpler approach consists of a generalization of the correlation operator technique [47, which has proven very useful in the variational theory of light nuclei, particularly in the context of variational Monte Carlo calculations [2.48]. In such an approach, known as the transition-correlation-operator (TCO) method [49], the nuclear wave function is written as 


$$
\Psi_{N+\Delta}=\left[\mathcal{S} \prod_{i<j}\left(1+U_{i j}^{T R}\right)\right] \Psi,
$$

where $\Psi$ is the purely nucleonic component, $\mathcal{S}$ is the symmetrizer and the transition operators $U_{i j}^{T R}$ convert $N N$ pairs into $N \Delta$ and $\Delta \Delta$ pairs. In the present study the $\Psi$ is taken from PHH solutions of the AV18/UIX Hamiltonian with nucleons only interactions [49], while the

$U_{i j}^{T R}$ is obtained from two-body bound and low-energy scattering state solutions of the full $N-\Delta$ coupled-channel problem. This aspect of the present calculations is reviewed briefly in the next subsection.

\section{A. Wave functions with $\Delta$-admixtures}

The transition correlation operator (TCO) method 49] consists in approximating the $\Psi_{N+\Delta}$ as in Eq. (3.3), with the transition operators $U_{i j}^{T R}$ defined as

$$
\begin{gathered}
U_{i j}^{T R}=U_{i j}^{N \Delta}+U_{i j}^{\Delta N}+U_{i j}^{\Delta \Delta}, \\
U_{i j}^{N \Delta}=\left[u^{\sigma \tau I I}\left(r_{i j}\right) \boldsymbol{\sigma}_{i} \cdot \mathbf{S}_{j}+u^{t \tau I I}\left(r_{i j}\right) S_{i j}^{I I}\right] \boldsymbol{\tau}_{i} \cdot \mathbf{T}_{j}, \\
U_{i j}^{\Delta \Delta}=\left[u^{\sigma \tau I I I}\left(r_{i j}\right) \mathbf{S}_{i} \cdot \mathbf{S}_{j}+u^{t \tau I I}\left(r_{i j}\right) S_{i j}^{I I I}\right] \mathbf{T}_{i} \cdot \mathbf{T}_{j} .
\end{gathered}
$$

Here, $\mathbf{S}_{i}$ and $\mathbf{T}_{i}$ are spin- and isospin-transition operators which convert nucleon $i$ into a $\Delta$-isobar; $S_{i j}^{I I}$ and $S_{i j}^{I I I}$ are tensor operators in which, respectively, the Pauli spin operators of either particle $i$ or $j$, and both particles $i$ and $j$ are replaced by corresponding spintransition operators. The $U_{i j}^{T R}$ vanishes in the limit of large interparticle separations, since no $\Delta$-components can exist asymptotically.

The transition operator $U_{i j}^{T R}$ and nucleonic wave function $\Psi$ in Eq. (3.3) could be determined variationally by using an interaction of the form given in Eq. (3.2), that contains both $N$ and $\Delta$ degrees of freedom, such as the Argonne $v_{28 Q}$ (AV28Q) model [7,50], and by minimizing the ground-state energy of each given nucleus. Instead, we use transition correlation functions $u^{\sigma \tau I I}(r)$, etc. (shown in Fig. 11) that approximately reproduce two-body bound- and low-energy scattering-state wave functions for the AV28Q model, and take the PHH wave function obtained in Sec. II-A as the $\Psi$ in Eq. (3.3). The validity of such an approximation has been discussed at length in the original reference [49]. Here, we only note that i) since the correlation functions $u^{\sigma \tau I I}(r)$, etc. are short-ranged, they are expected to have a rather weak dependence on $A$; ii) it is important the $\Psi$ used in Eq. (3.3), obtained from a $v_{i j}(N N \rightarrow N N)$ interaction phase-equivalent to the full $v_{i j}$ of Eq. (3.2), be proportional to that projected out from the $\Psi_{N+\Delta}$ wave function for the $v_{i j}$ interaction. This has been explicitly verified by direct calculation in the two-body problem [49].

In the TCO scheme, the perturbation theory description of $\Delta$-admixtures is equivalent to the replacements:

$$
\begin{aligned}
& U_{i j}^{N \Delta, \mathrm{PT}}=\frac{v_{i j}(N N \rightarrow N \Delta)}{m-m_{\Delta}}, \\
& U_{i j}^{\Delta \Delta, \mathrm{PT}}=\frac{v_{i j}(N N \rightarrow \Delta \Delta)}{2\left(m-m_{\Delta}\right)},
\end{aligned}
$$


where the kinetic energy contributions in the denominators of Eqs. (3.7) and (3.8) have been neglected (static $\Delta$ approximation). The transition interactions $v_{i j}(N N \rightarrow N \Delta)$ and $v_{i j}(N N \rightarrow \Delta \Delta)$ have the same operator structure as $U_{i j}^{N \Delta}$ and $U_{i j}^{\Delta \Delta}$ of Eqs. (3.5) and (3.6), but with the $u^{\sigma \tau \alpha}(r)$ and $u^{t \tau \alpha}(r)$ functions replaced by, respectively,

$$
\begin{aligned}
& v^{\sigma \tau \alpha}(r)=\frac{(f f)_{\alpha}}{4 \pi} \frac{m_{\pi}}{3} \frac{e^{-x}}{x} C(x), \\
& v^{t \tau \alpha}(r)=\frac{(f f)_{\alpha}}{4 \pi} \frac{m_{\pi}}{3}\left(1+\frac{3}{x}+\frac{3}{x^{2}}\right) \frac{e^{-x}}{x} C^{2}(x) .
\end{aligned}
$$

Here $\alpha=$ II, III, $x \equiv m_{\pi} r$, $(f f)_{\alpha}=f_{\pi N N} f_{\pi N \Delta}, f_{\pi N \Delta} f_{\pi N \Delta}$ for $\alpha=$ II, III, respectively, and the cutoff function $C(x)=1-e^{-\lambda x^{2}}, \lambda=4.09$ in the AV28Q model [50]. Note that in Fig. $11 u^{q \mathrm{II}, \mathrm{PT}}(r)=v^{q \mathrm{II}}(r) /\left(m-m_{\Delta}\right)$ and $u^{q \mathrm{III}, \mathrm{PT}}(r)=v^{q \mathrm{III}}(r) /\left[2\left(m-m_{\Delta}\right)\right]$, with $q=\sigma \tau$, $t \tau$.

The perturbative treatment has been often (in fact, almost exclusively) used in the literature to estimate the effect of $\Delta$ degrees of freedom on electroweak observables. However, it may lead to a substantial overprediction of their importance [18,49], since it produces $N \Delta$ and $\Delta \Delta$ wave functions which are too large at short distance, see Fig. 11 .

\section{B. $N \Delta$-transition and $\Delta$ currents}

The nuclear electromagnetic current is expanded into a sum of many-body terms that operate on the nucleon and $\Delta$-isobar degrees of freedom. The nucleonic component of this current operator has been discussed in the previous section. Here, we only discuss its $\Delta$ components.

\section{1. $N \Delta$-transition and $\Delta$ one-body currents}

The one-body current is written as

$$
\mathbf{j}_{i}^{(1)}(\mathbf{q})=\sum_{B, B^{\prime}=N, \Delta} \mathbf{j}_{i}\left(\mathbf{q} ; B \rightarrow B^{\prime}\right)
$$

where $\mathbf{j}_{i}(\mathbf{q} ; N \rightarrow N)$ is the nucleonic current component given in Eq. (2.13) and

$$
\begin{aligned}
& \mathbf{j}_{i}(\mathbf{q} ; N \rightarrow \Delta)=-\frac{\mathrm{i}}{2 \mathrm{~m}} G_{\gamma N \Delta}\left(q_{\mu}^{2}\right) e^{\mathrm{i} \mathbf{q} \cdot \mathbf{r}_{i}} \mathbf{q} \times \mathbf{S}_{i} T_{z, i}, \\
& \mathbf{j}_{i}(\mathbf{q} ; \Delta \rightarrow \Delta)=-\frac{\mathrm{i}}{24 \mathrm{~m}} G_{\gamma \Delta \Delta}\left(q_{\mu}^{2}\right) e^{\mathrm{i} \mathbf{q} \cdot \mathbf{r}_{i}} \mathbf{q} \times \boldsymbol{\Sigma}_{i}\left(1+\Theta_{z, i}\right) .
\end{aligned}
$$

Here $\boldsymbol{\Sigma}(\boldsymbol{\Theta})$ is the Pauli operator for the $\Delta$ spin $3 / 2$ (isospin 3/2), and the expression for $\mathbf{j}_{i}(\mathbf{q} ; \Delta \rightarrow N)$ is obtained from that for $\mathbf{j}_{i}(\mathbf{q} ; N \rightarrow \Delta)$ by replacing the transition spin and isospin operators by their hermitian conjugates. The $N \Delta$-transition and $\Delta$ electromagnetic form factors, respectively $G_{\gamma N \Delta}$ and $G_{\gamma \Delta \Delta}$, are parametrized as 


$$
\begin{aligned}
G_{\gamma N \Delta}\left(q_{\mu}^{2}\right) & =\frac{\mu_{\gamma N \Delta}}{\left(1+q_{\mu}^{2} / \Lambda_{N \Delta, 1}^{2}\right)^{2} \sqrt{1+q_{\mu}^{2} / \Lambda_{N \Delta, 2}^{2}}}, \\
G_{\gamma \Delta \Delta}\left(q_{\mu}^{2}\right) & =\frac{\mu_{\gamma \Delta \Delta}}{\left(1+q_{\mu}^{2} / \Lambda_{\Delta \Delta}^{2}\right)^{2}} .
\end{aligned}
$$

Here the $N \Delta$-transition magnetic moment $\mu_{\gamma N \Delta}$ is taken equal to $3 \mu_{N}$, as obtained from an analysis of $\gamma N$ data in the $\Delta$-resonance region [51]; this analysis also gives $\Lambda_{N \Delta, 1}=$ $0.84 \mathrm{GeV}$ and $\Lambda_{N \Delta, 2}=1.2 \mathrm{GeV}$. The value used for the $\Delta$ magnetic moment $\mu_{\gamma \Delta \Delta}$ is $4.35 \mu_{N}$ by averaging results of a soft-photon analysis of pion-proton bremsstrahlung data near the $\Delta^{++}$resonance [52], and $\Lambda_{\Delta \Delta}=0.84 \mathrm{GeV}$ as in the dipole parametrization of the nucleon form factor. In principle, $N$ to $\Delta$ excitation can also occur via an electric quadrupole transition. Its contribution, however, has been ignored, since the associated pion photoproduction amplitude is found to be experimentally small at resonance [53]. Also neglected is the $\Delta$ convection current.

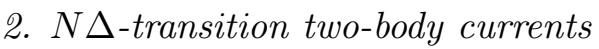

The two-body term is written as

$$
\mathbf{j}_{i j}^{(2)}(\mathbf{q})=\sum_{B_{i}, B_{j}=N, \Delta}^{\prime} \sum_{B_{i}^{\prime}, B_{j}^{\prime}=N, \Delta}^{\prime} \mathbf{j}_{i j}\left(\mathbf{q} ; B_{i} B_{j} \rightarrow B_{i}^{\prime} B_{j}^{\prime}\right),
$$

where the prime over the summation symbols indicates that terms involving more than a single $\Delta$ have been neglected in the present study. The $N N \rightarrow N N$ two-body terms have already been discussed. The two-body terms involving at most a single $\Delta$ are illustrated in Fig. 12, and are explicitly given by

$$
\begin{aligned}
\mathbf{j}_{i j}(\mathbf{q} ; N N \rightarrow N \Delta) & =\left(\boldsymbol{\tau}_{i} \times \mathbf{T}_{j}\right)_{z}\left[\left[\boldsymbol{\sigma}_{i}\left(\mathbf{S}_{j} \cdot \hat{\mathbf{r}}_{i j}\right) e^{\mathrm{i} \mathbf{q} \cdot \mathbf{r}_{i}}+\left(\boldsymbol{\sigma}_{i} \cdot \hat{\mathbf{r}}_{i j}\right) \mathbf{S}_{j} e^{\mathrm{i} \mathbf{q} \cdot \mathbf{r}_{j}}\right] h\left(r_{i j}\right)\right. \\
& \left.+e^{\mathbf{i q} \cdot \mathbf{R}_{i j}}\left(\boldsymbol{\sigma}_{i} \cdot \boldsymbol{\nabla}_{i}\right)\left(\mathbf{S}_{j} \cdot \boldsymbol{\nabla}_{j}\right) \hat{\mathbf{r}}_{i j} \bar{h}\left(r_{i j}\right)\right]
\end{aligned}
$$

where $\mathbf{r}_{i j}=\mathbf{r}_{i}-\mathbf{r}_{j}, \mathbf{R}_{i j}=\left(\mathbf{r}_{i}+\mathbf{r}_{j}\right) / 2$, and the functions $h(r)$ and $\bar{h}(r)$ are defined as, respectively,

$$
\begin{aligned}
& h(r) \equiv-\left(\frac{f_{\pi N N} f_{\pi N \Delta}}{4 \pi}\right) \frac{1}{x^{2}}(1+x) e^{-x}, \\
& \bar{h}(r) \equiv\left(\frac{f_{\pi N N} f_{\pi N \Delta}}{4 \pi}\right) \frac{1}{m_{\pi}^{2}} \int_{-\frac{1}{2}}^{+\frac{1}{2}} d z e^{-\mathbf{i} z \mathbf{q} \cdot \mathbf{r}} e^{-r L(z)},
\end{aligned}
$$

with $x=m_{\pi} r$ and $L(z)=\left[m_{\pi}^{2}+q^{2}\left(1 / 4-z^{2}\right)\right]^{1 / 2}$. Terms explicitly proportional to $\mathbf{q}$ in Eq. (3.17) have been dropped, since in applications only the transverse components of $\mathbf{j}(\mathbf{q})$ occur. The three terms in Eq. (3.17) are associated with diagrams (a), (b) and (c) in Fig. 12, respectively, and can be obtained from the well known expression of the two-body nucleonic currents due to pion-exchange by replacing $\boldsymbol{\sigma}_{j}$ and $\boldsymbol{\tau}_{j}$ with $\mathbf{S}_{j}$ and $\mathbf{T}_{j}$, respectively. 
To account for the hadron extended structure, form factors must be introduced at the $\pi N N$ and $\pi N \Delta$ vertices. In the case of $v_{i j}(N N \rightarrow N \Delta)$ interaction, an $r$-space gaussian cutoff has been used. However, for the $j(N N \rightarrow N \Delta)$ above it has been found convenient to introduce monopole form factors with $\Lambda=900 \mathrm{MeV}$ in its $p$-space expression. This value for $\Lambda$ is consistent with that obtained from the tensor component of $v_{i j}(N N \rightarrow N \Delta)$. Finally, the expression in Eq. (3.17) is multiplied by the isovector form factor $G_{E}^{V}\left(q_{\mu}^{2}\right)$.

\section{Calculation}

Calculation of the magnetic form factors requires evaluation of the transition matrix element in Eq. (2.28), where the wave functions and currents include both nucleonic and $\Delta$-isobar degrees of freedom. To evaluate such a matrix element, it is convenient to expand the wave function $\Psi_{N+\Delta, J_{z}}$ as

$$
\Psi_{N+\Delta, J_{z}}=\Psi_{J_{z}}+\sum_{i<j} U_{i j}^{T R} \Psi_{J_{z}}+\ldots
$$

and write the numerator of Eq. (2.28), in a schematic notation, as

$$
\left\langle\Psi_{N+\Delta, f}|j| \Psi_{N+\Delta, i}\right\rangle=\left\langle\Psi_{f}\right| j(N \text { only })\left|\Psi_{i}\right\rangle+\left\langle\Psi_{f}|j(\Delta)| \Psi_{i}\right\rangle,
$$

where $j(N$ only) denotes all one- and two-body contributions to $\mathbf{j}(\mathbf{q})$ which only involve nucleon degrees of freedom, i.e., $j(N$ only $)=j^{(1)}(N \rightarrow N)+j^{(2)}(N N \rightarrow N N)$. The operator $j(\Delta)$ includes terms involving the $\Delta$-isobar degrees of freedom, associated with the explicit $\Delta$ currents $j^{(1)}(N \rightarrow \Delta), j^{(1)}(\Delta \rightarrow N), j^{(1)}(\Delta \rightarrow \Delta), j^{(2)}(N N \rightarrow N \Delta)$, etc., and with the transition operators $U_{i j}^{T R}$. The operator $j(\Delta)$ is illustrated diagrammatically in Figs. 13 and 14. The terms (a)-(g) in Fig. 13 and (a)-(f) in Fig. 14 are two-body current operators. The terms (g)-(l) in Fig. 14 are three-body current operators, while the terms (h)(j) in Fig. 13 are to be interpreted as renormalization corrections to the "nucleonic" matrix elements $\left\langle\Psi_{f}\right| j$ (Nonly) $\left|\Psi_{i}\right\rangle$, due to the presence of $\Delta$-admixtures in the wave functions.

There are, however, additional, connected three-body terms in $j(\Delta)$ that are neglected in the present work. A number of these are illustrated in Fig. 15. Their contribution is expected to be significantly smaller than that from the terms in Figs. 13 and 14 involving transition correlations between two particles only, of the type $U_{i j}^{B B^{\prime \dagger}} U_{i j}^{B B^{\prime}}$, but comparable to that from the three-body terms in Fig. 14 having $U_{i j}^{B B^{\prime \dagger}} U_{j k}^{B B^{\prime}}$. These have been found to be very small.

The terms in Fig. 13 are expanded as operators acting on the nucleons' coordinates. For example, the terms (a) and (e) in Fig. [13] have the structure, respectively,

$$
\begin{aligned}
& \text { (a) }=j_{i}^{(1)}(\Delta \rightarrow N) U_{i j}^{\Delta N}, \\
& \text { (e) }=U_{i j}^{\Delta N^{\dagger}} j_{i}^{(1)}(\Delta \rightarrow \Delta) U_{i j}^{\Delta N},
\end{aligned}
$$

which can be reduced to operators involving only Pauli spin and isospin matrices by using the identities 


$$
\begin{aligned}
\mathbf{S}^{\dagger} \cdot \mathbf{A ~ S} \cdot \mathbf{B}= & \frac{2}{3} \mathbf{A} \cdot \mathbf{B}-\frac{\mathrm{i}}{3} \boldsymbol{\sigma} \cdot(\mathbf{A} \times \mathbf{B}) \\
\mathbf{S}^{\dagger} \cdot \mathbf{A} \boldsymbol{\Sigma} \cdot \mathbf{B} \mathbf{S} \cdot \mathbf{C}= & \frac{5}{3} \mathrm{i} \mathbf{A} \cdot(\mathbf{B} \times \mathbf{C})-\frac{1}{3} \boldsymbol{\sigma} \cdot \mathbf{A} \mathbf{B} \cdot \mathbf{C} \\
& -\frac{1}{3} \mathbf{A} \cdot \mathbf{B} \mathbf{C} \cdot \boldsymbol{\sigma}+\frac{4}{3} \mathbf{A} \cdot(\mathbf{B} \cdot \boldsymbol{\sigma}) \mathbf{C}
\end{aligned}
$$

where $\mathbf{A}, \mathbf{B}$ and $\mathbf{C}$ are vector operators that commute with $\boldsymbol{\sigma}$, but not necessarily among themselves.

While the terms in Fig. 14 could have been reduced in precisely the same way, the resulting expressions in terms of $\boldsymbol{\sigma}$ and $\boldsymbol{\tau}$ Pauli matrices become too cumbersome. Thus, for these it was found to be more convenient to retain the explicit representation of $\mathbf{S}\left(\mathbf{S}^{\dagger}\right)$ as a $4 \times 2(2 \times 4)$ matrix

$$
\mathbf{S}=\left(\begin{array}{cc}
-\hat{\mathbf{e}}_{-} & 0 \\
\sqrt{\frac{2}{3}} \hat{\mathbf{e}}_{0} & -\frac{1}{\sqrt{3}} \hat{\mathbf{e}}_{-} \\
-\frac{1}{\sqrt{3}} \hat{\mathbf{e}}_{+} & \sqrt{\frac{2}{3}} \hat{\mathbf{e}}_{0} \\
0 & -\hat{\mathbf{e}}_{+}
\end{array}\right),
$$

where $\hat{\mathbf{e}}_{ \pm}=\mp(\hat{\mathbf{x}} \pm \mathrm{i} \hat{\mathbf{y}}) / \sqrt{2}, \hat{\mathbf{e}}_{0}=\hat{\mathbf{z}}$, and $\hat{\mathbf{e}}_{\mu}^{*}=(-)^{\mu} \hat{\mathbf{e}}_{-\mu}$ and derive the result of terms such as $(\mathrm{a})+(\mathrm{c})+(\mathrm{e})=U_{i j}^{N \Delta^{\dagger}} j_{i j}^{(2)}(N N \rightarrow N \Delta)$ on the state $|\Psi\rangle$ by first operating with $j^{(2)}$ and then with $U^{N \Delta^{\dagger}}$. The Monte Carlo evaluation of the matrix element is then performed with methods similar to those sketched in Sec. II-D.

The normalization of the wave function is given by

$$
\begin{aligned}
\left\langle\Psi_{N+\Delta, J_{z}} \mid \Psi_{N+\Delta, J_{z}}\right\rangle & =\left\langle\Psi_{J_{z}}\left|1+\sum_{i<j}\left[2 U_{i j}^{\Delta N^{\dagger}} U_{i j}^{\Delta N}+U_{i j}^{\Delta \Delta{ }^{\dagger}} U_{i j}^{\Delta \Delta}\right]\right| \Psi_{J_{z}}\right\rangle \\
& +(\text { three-body terms })
\end{aligned}
$$

and the three-body terms have been neglected consistently with the approximation introduced in Eq. (3.21), as discussed above.

Perturbation theory (PT) estimates of the importance of $\Delta$-isobar degrees of freedom in photo- and electro-nuclear observables typically include only the contribution from single $N \rightleftharpoons \Delta$ transitions (namely diagrams (a) and (b) in Fig. 13) and ignore the change in the wave function normalization. In the TCO scheme, these PT estimates are obtained by using $U^{B B^{\prime}, P T}$ transition correlation defined in Eqs. (3.7) and 3.8) 49. In particular, the PT expressions for the three-body terms in Fig. 14, diagrams (g)-(h)along with those in which the first and third legs are exchanged, can easily be shown to satisfy current conservation with the Fujita-Miyazawa two-pion exchange three-nucleon interaction (TNI) [54] given by

$$
V_{i j k}^{F M}(N N N \rightarrow N N N)=v_{j k}(\Delta N \rightarrow N N) \frac{1}{m-m_{\Delta}} v_{i j}(N N \rightarrow N \Delta)+\text { h.c. }
$$

where the transition potentials are defined in Eqs. (3.9) and (3.10) (here with the cutoff function $C(x)$ set to one). Current models of TNI [⿴囗十 include the "long-range" $2 \pi$-exchange component above. Indeed, the need of including the associated three-body currents provided one of the motivations for undertaking the present study. 


\section{The magnetic moments and form factors}

The ${ }^{3} \mathrm{H}$ and ${ }^{3} \mathrm{He}$ magnetic form factors obtained by including nucleon and $\Delta$-isobar degrees of freedom in the nuclear wave functions and currents are shown in Figs. 16 and

17; while individual contributions to the combinations $F_{M}^{S}$ and $F_{M}^{V}$ are displayed in Figs. 18 and 19. Finally, individual and cumulative contributions to the magnetic moments and cumulative contributions to the magnetic radii of the trinucleons are listed in Tables [1, III and $\mathbb{\square}$, respectively. Note that in Figs. 16 and 17 and Table $\mathbb{M}$ the contributions labelled 1- $\Delta$ and 2- $\Delta$ are associated with the diagrams in Figs. 13 and 14, respectively. Also note that the individual nucleonic and $\Delta$-isobar contributions in Figs. 18 and 19 and Table $\square$ are normalized as, in a schematic notation,

$$
[O]=\frac{\left\langle\Psi\left|j_{O}\right| \Psi\right\rangle}{\langle\Psi \mid \Psi\rangle} .
$$

However, the cumulative contributions in Figs. 16 and 17 and Table [II] and IV are normalized as

$$
[\text { TOT }-\mathrm{N}]=\frac{\langle\Psi| j(N \text { only })|\Psi\rangle}{\langle\Psi \mid \Psi\rangle}
$$

when "nucleons only" terms are retained, and as

$$
[\mathrm{TOT}-(\mathrm{N}+\Delta)]=\frac{\left\langle\Psi_{N+\Delta}|j(N+\Delta)| \Psi_{N+\Delta}\right\rangle}{\left\langle\Psi_{N+\Delta} \mid \Psi_{N+\Delta}\right\rangle},
$$

when, in addition, the $\Delta$ terms are included.

The contributions associated with $\Delta$-components are found to be small in contrast to earlier studies [55]. In particular, we find that the sign of the 2- $\Delta$ contribution in Fig. [19 is opposite to that reported in Ref. [55]. The origin of this difference is unclear at this point. However, we do find that the sign of the $2-\Delta$ contribution (see Fig. 19) is the same as that of the nucleonic PS ( $\pi$-like) contribution, as one would expect.

The predicted magnetic moments of the trinucleons are within less than $1 \%$ of the experimental values. The predominatly isovector $\Delta$-isobar contributions lead to an increase (in magnitude) of the ${ }^{3} \mathrm{H}$ and ${ }^{3} \mathrm{He}$ magnetic moments calculated with nucleons only degrees of freedom of, respectively, $1.1 \%$ and $1.7 \%$ in relative terms. We note that perturbation theory estimates of the $\Delta$-isobar contributions are found to be significantly larger than obtained here [5].

The predicted magnetic radii of ${ }^{3} \mathrm{H}$ and ${ }^{3} \mathrm{He}$ are, respectively, $2 \%$ and $3 \%$ smaller than the experimental values, but still within experimental errors. Inclusion of the contributions due to two- and three-body exchange currents leads to a decrease of the ${ }^{3} \mathrm{H}$ and ${ }^{3} \mathrm{He}$ magnetic radii of, respectively, $5 \%$ and $6 \%$.

While the agreement between theory and experiment is satisfactory for the magnetic moments, magnetic radii and low $q$ form factors, the calculated form factors, particularly that of ${ }^{3} \mathrm{He}$, remain at variance with the experiment in the diffraction region. The role played by $\Delta$-isobar degrees of freedom is found to be marginal over the whole $q$-range considered here. 


\section{CONCLUSIONS}

The present results for the electromagnetic form factors of the trinucleons may be summarized as follows: i) the trinucleon charge form factors agree well with the experimental values when calculated with wave functions obtained from a Hamiltonian consisting of the Argonne $v_{18}$ two-nucleon and the Urbana IX three-nucleon interactions; ii) agreement with the experimental charge form factors requires that the two-nucleon exchange charge operators are taken into account; iii) the calculated magnetic form factor of ${ }^{3} \mathrm{H}$ agrees well with experiment, whereas that of ${ }^{3} \mathrm{He}$ agrees well with the experimental values only for momentum transfer values below the first zero in the form factor; iv) the two-nucleon exchange current contributions are essential for achieving agreement with experiment whereas threenucleon exchange current operators and the $\Delta$-isobar configurations have only very small effects on the calculated magnetic form factors.

The result for the static observables are that the calculated value for the isovector combination of the trinucleon magnetic moments agrees completely with the experimental value (Table [II). The isoscalar combination of the trinucleon magnetic moments exceeds the experimental value by about $5 \%$, but this small disagreement does not prevent a good reproduction of the isoscalar combination of the experimental magnetic form factors. As the calculated magnetic moments of ${ }^{3} \mathrm{H}$ and ${ }^{3} \mathrm{He}$ differ by less than $0.015 \mathrm{n} . \mathrm{m}$. from their experimental values, the results appear to be very satisfactory. The calculated charge radii are smaller by only $2 \%$ than the experimental values. The calculated magnetic radii are smaller than $3 \%$ than the experimental values (Tables $[\mathrm{V}$ and $\mathrm{V}$ ). To obtain these quite satisfactory calculated values for the charge and magnetic radii the exchange current contributions have to be taken into account.

We note finally that the three-nucleon exchange current operator (2.24)-(2.26), which was constructed to satisfy the continuity equation with the three-nucleon interaction (2.19), was found to give only very small contributions to the magnetic form factors of the trinucleons. It is worth noting that the corresponding component of the "Tucson-Melbourne" type three-nucleon interaction in [30] is roughly an order of magnitude stronger than the three-nucleon interaction (2.19), and would therefore imply correspondingly much larger three-nucleon exchange current contributions. Part of this difference is the inclusion of $\Delta$-isobar intermediate state effects in the "d" term of that three-nucleon interaction. In the present work $\Delta$-isobar configurations are treated explicitly, and should therefore not be included in irreducible three-nucleon exchange current operators.

\section{ACKNOWLEDGMENTS}

We wish to thank A. Kievsky, S. Rosati and M. Viviani for letting us use their PHH wave functions, and I. Sick for providing us with tables of the experimental data. The support of the U.S. Department of Energy via a graduate research assistantship provided by Jefferson Lab is gratefully acknowledged by L.E.M. The work of D.O.R. is partially supported by the Academy of Finland under contract 34081 while that of R.S. is supported by the U.S. Department of Energy. Finally, the calculations were made possible by grants of time from the National Energy Research Supercomputer Center in Livermore. 


\section{REFERENCES}

[1] J. Carlson and R. Schiavilla, preprint JLAB-THY-97-20, Rev. Mod. Phys. in press (1998).

[2] B.S. Pudliner, V.R. Pandharipande, J. Carlson, S.C. Pieper, and R.B. Wiringa, Phys. Rev. C 56, 1720 (1997).

[3] R.B. Wiringa, V.G.J. Stoks, and R. Schiavilla, Phys. Rev. C 51, 38 (1995).

[4] B.S. Pudliner, V.R. Pandharipande, J. Carlson, and R.B. Wiringa, Phys. Rev. Lett. 74, 4396 (1995).

[5] R. Schiavilla, V.R. Pandharipande, and D.O. Riska, Phys. Rev. C 40, 2294 (1989).

[6] R. Schiavilla, V.R. Pandharipande, and D.O. Riska, Phys. Rev. C 41, 309 (1990).

[7] R.B. Wiringa, R.A. Smith, and T.L. Ainsworth, Phys. Rev. C 29, 1207 (1984).

[8] M.T. Peña, H. Henning and P.U. Sauer, Phys. Rev. C 42, 855 (1990).

[9] R.B. Wiringa, V. Ficks, and A. Fabrocini, Phys. Rev. C 38, 1010 (1988).

[10] A. Kievsky, S. Rosati, and M. Viviani, Nucl. Phys. A551, 241 (1993).

[11] A. Kievsky, M. Viviani, and S. Rosati, Nucl. Phys. A577, 511 (1994).

[12] A. Kievsky, M. Viviani, and S. Rosati, Phys. Rev. C 52, R15 (1995).

[13] M. Viviani, A. Kievsky, and S. Rosati, Few-Body Syst. 18, 25 (1995).

[14] W. Glöckle, The Quantum Mechanical Few-Body Problem (Springer-Verlag, BerlinHeiderberg, 1983).

[15] C.R. Chen, C.L. Payne, J.L. Friar, and B.F. Gibson, Phys. Rev. C 33, 1740 (1986).

[16] W. Glöckle et al., Few Body Syst. Suppl. 8, 9 (1995).

[17] D.O. Riska, Phys. Rep. 181, 207 (1989).

[18] M. Viviani, R. Schiavilla, and A. Kievsky, Phys. Rev. C 54, 534 (1996).

[19] H. Berg et al., Nucl. Phys. A334, 21 (1980).

[20] M. Chemtob and M. Rho, Nucl. Phys. A163, 1 (1971); A212, 628 (1973).

[21] J. Carlson, V.R. Pandharipande, and R. Schiavilla, Modern Topics in Electron Scattering, B. Frois and I. Sick, eds. (World Scientific, Singapore, 1991), p. 177.

[22] D.O. Riska, Phys. Scr. 31, 471 (1985).

[23] J. Carlson, D.O. Riska, R. Schiavilla, and R.B. Wiringa, Phys. Rev. C 42, 830 (1990).

[24] R. Schiavilla and D.O. Riska, Phys. Rev. C 43, 437 (1991).

[25] J.L. Friar, Ann. Phys. 104, 380 (1977).

[26] S.A. Coon and J.L. Friar, Phys. Rev. C 34, 1060 (1986).

[27] F. Coester and D.O. Riska, Ann. Phys. 234, 141 (1994).

[28] R. Schiavilla, to be published.

[29] S. Weinberg, Phys. Rev. Lett. 18, 188 (1967).

[30] S.A. Coon and M.T. Peña, Phys. Rev. C 48, 2559 (1993).

[31] N. Metropolis et al., J. Chem. Phys. 21, 1087 (1953).

[32] H. Collard et al., Phys. Rev. 138, 357 (1965).

[33] J.S. McCarthy, I. Sick, and R. Whitney, Phys. Rev. C 15, 1396 (1977).

[34] Z.M. Szalata et al., Phys. Rev. C 15, 1200 (1977).

[35] R.G. Arnold et al., Phys. Rev. Lett. 40, 1429 (1978).

[36] J.M. Cavedon et al., Phys. Rev. Lett. 49, 978 (1982).

[37] P.C. Dunn et al., Phys. Rev. C 27, 71 (1983).

[38] C.R. Ottermann et al., Nucl. Phys. A435, 688 (1985).

[39] F.P. Juster et al., Phys. Rev. Lett. 55, 2261 (1985).

[40] D.H. Beck et al., Phys. Rev. Lett. 59, 1537 (1987). 
[41] A. Amroun et al., Nucl. Phys. A579, 596 (1994).

[42] J. Hockert, D.O. Riska, M. Gari, and A. Huffman, Nucl. Phys. A217, 14 (1973).

[43] M. Gari and W. Krümpelmann, Phys. Lett. B173, 10 (1986).

[44] R. Schiavilla, Perspectives in Nuclear Physics at Intermediate Energies, S. Boffi, C. Ciofi degli Atti, and M. Giannini, eds., (World Scientific, Singapore, 1996).

[45] Ch. Hajduk, P.U. Sauer, and W. Strueve, Nucl. Phys. A405, 581 (1983).

[46] A. Picklesimer, R.A. Rice, and R. Brandenburg, Phys. Rev. Lett. 68, 1484 (1992).

[47] J. Lomnitz-Adler, V.R. Pandharipande, and R.A. Smith, Nucl. Phys. A361, 399 (1981).

[48] R.B. Wiringa, Phys. Rev. C 43, 1585 (1991).

[49] R. Schiavilla, R.B. Wiringa, V.R. Pandharipande, and J. Carlson, Phys. Rev. C 45, 2628 (1992).

[50] R.B. Wiringa, private comunication.

[51] C.E. Carlson, Phys. Rev. D 34, 2704 (1986).

[52] D. Lin and M.K. Liou, Phys. Rev. C 43, R930 (1991).

[53] T.E.O. Ericson and W. Weise, Pions and Nuclei (Clarendon Press, Oxford, 1988).

[54] J. Fujita and H. Miyazawa, Prog. Theor. Phys. 17, 360 (1957).

[55] W. Strueve, Ch. Hajduk, P.U. Sauer, and W. Theis, Nucl. Phys. A465, 651 (1986). 


\section{TABLES}

TABLE I. Binding energies corresponding to the AV14 and AV18/UIX Hamiltonian models. The AV14 results obtained with the PHH expansion are compared with those calculated by solving the Faddeev equations in configuration $(\mathrm{F} / \mathrm{R})$ and in momentum $(\mathrm{F} / \mathrm{P})$ space. The statistical error associated with the GFMC calculations are shown in parenthesis.

\begin{tabular}{llll}
\hline \hline Model & Method & $\mathrm{B}\left({ }^{3} \mathrm{H}\right)(\mathrm{MeV})$ & $\mathrm{B}\left({ }^{3} \mathrm{He}\right)(\mathrm{MeV})$ \\
\hline \multirow{2}{*}{ AV14 } & PHH & 7.683 & 7.032 \\
& F/R & 7.670 & 7.014 \\
& F/P & 7.680 & 7.75 \\
\hline AV18/UIX & PHH & 8.49 & $7.71(1)$ \\
\hline & GFMC & $8.47(1)$ & 7.72 \\
\hline
\end{tabular}

TABLE II. Individual contributions from the different components of the nuclear electromagnetic current operator to the ${ }^{3} \mathrm{H}$ and ${ }^{3} \mathrm{He}$ magnetic moments and their $\mu_{S}$ and $\mu_{V}$ combinations, in nuclear magnetons (n.m.). Note that, because of isospin-symmetry breaking components present in the $\mathrm{PHH}{ }^{3} \mathrm{H}$ and ${ }^{3} \mathrm{He}$ wave functions, purely isoscalar (isovector) currents give non vanishing contributions to the $\mu_{V}\left(\mu_{S}\right)$ combination. The contributions to $\mu_{S}$ due to the $\pi \pi_{S}$ and $2-\Delta$ currents and those to $\mu_{V}$ due to the SO2+LL currents are very small and are not listed.

\begin{tabular}{lrrrr}
\hline \hline & $\mu\left({ }^{3} \mathrm{H}\right)$ & $\mu\left({ }^{3} \mathrm{He}\right)$ & $\mu_{S}$ & $\mu_{V}$ \\
\hline $1-\mathrm{N}$ & 2.571 & -1.757 & 0.407 & 2.164 \\
$\mathrm{PS}$ & 0.274 & -0.269 & 0.002 & 0.271 \\
$\mathrm{~V}$ & 0.046 & -0.044 & 0.001 & 0.045 \\
$\mathrm{SO}$ & 0.057 & 0.010 & 0.033 & 0.023 \\
$\mathrm{SO} 2+\mathrm{LL}$ & -0.005 & -0.006 & -0.005 & \\
$\rho \pi \gamma+\omega \pi \gamma$ & 0.016 & -0.009 & 0.003 & 0.012 \\
$\pi \pi_{S}$ & 0.002 & -0.002 & & 0.002 \\
$1-\Delta$ & 0.084 & -0.064 & 0.010 & 0.074 \\
$2-\Delta$ & 0.024 & -0.024 & 0.024 \\
\hline \hline
\end{tabular}

TABLE III. Cumulative and normalized contributions to the ${ }^{3} \mathrm{H}$ and ${ }^{3} \mathrm{He}$ magnetic moments and their $\mu_{S}$ and $\mu_{V}$ combinations, in nuclear magnetons (n.m.), compared with the experimental data.

\begin{tabular}{lcccr}
\hline \hline & $\mu\left({ }^{3} \mathrm{H}\right)$ & $\mu\left({ }^{3} \mathrm{He}\right)$ & $\mu_{S}$ & $\mu_{V}$ \\
\hline $1-\mathrm{N}$ & 2.571 & -1.757 & 0.407 & 2.164 \\
TOT-N & 2.961 & -2.077 & 0.442 & 2.519 \\
TOT-N+1- $\Delta$ & 2.971 & -2.089 & 0.441 & 2.530 \\
TOT- $(\mathrm{N}+\Delta)$ & 2.994 & -2.112 & 0.441 & 2.553 \\
EXP & 2.979 & -2.127 & 0.426 & 2.553 \\
\hline \hline
\end{tabular}


TABLE IV. Cumulative and normalized contributions to the ${ }^{3} \mathrm{H}$ and ${ }^{3} \mathrm{He}$ r.m.s. magnetic radii, in $\mathrm{fm}$, compared with the experimental data.

\begin{tabular}{lcc}
\hline \hline & ${ }^{3} \mathrm{H}$ & ${ }^{3} \mathrm{He}$ \\
\hline $1-\mathrm{N}$ & 1.895 & 2.040 \\
TOT-N & 1.810 & 1.925 \\
TOT-N $+1-\Delta$ & 1.804 & 1.916 \\
TOT- $(\mathrm{N}+\Delta)$ & 1.800 & 1.909 \\
EXP & $1.840 \pm 0.181$ & $1.965 \pm 0.153$ \\
\hline \hline
\end{tabular}

TABLE V. Cumulative and normalized contributions to the ${ }^{3} \mathrm{H}$ and ${ }^{3} \mathrm{He}$ r.m.s. charge radii, in fm, compared with the experimental data.

\begin{tabular}{lcc}
\hline \hline & ${ }^{3} \mathrm{H}$ & ${ }^{3} \mathrm{He}$ \\
\hline $1-\mathrm{N}$ & 1.711 & 1.919 \\
TOT & 1.725 & 1.928 \\
$\mathrm{EXP}$ & $1.755 \pm 0.086$ & $1.959 \pm 0.030$ \\
\hline \hline
\end{tabular}




\section{FIGURES}

FIG. 1. The magnetic form factors of ${ }^{3} \mathrm{H}$, obtained with single-nucleon currents $(1-\mathrm{N})$, and with inclusion of two-nucleon current $[(1+2)-\mathrm{N}]$ and $\pi \pi_{S}$ three-nucleon [TOT-N(D)] current contributions, are compared with data (shaded area) from Amroun et al. [41]. Theoretical results correspond to the AV18/UIX PHH wave functions, and employ the dipole parametrization (including the Galster factor for $\left.G_{E}\left(q_{\mu}^{2}\right)\right)$ for the nucleon electromagnetic form factors. Note that the Sachs form factor $G_{E}^{V}\left(q_{\mu}^{2}\right)$ is used in the model-independent isovector two-body currents obtained from the charge-independent part of the AV18 interaction. Also shown are the total results corresponding to the Gari-Krümpelmann parametrization [43] of the nucleon electromagnetic form factor $[\operatorname{TOT}-\mathrm{N}(\mathrm{GK})]$.

FIG. 2. Same as in Fig. 1, but for ${ }^{3} \mathrm{He}$.

FIG. 3. Individual contributions to the $F_{M}^{S}\left(q_{\mu}\right)$ combination, Eq. (2.30), of the ${ }^{3} \mathrm{H}$ and ${ }^{3} \mathrm{He}$ magnetic form factors, obtained with the dipole parametrization of the nucleon electromagnetic form factors. The sign of each contribution is given in parenthesis. Note that, because of isospin-symmetry breaking components present in the ${ }^{3} \mathrm{H}$ and ${ }^{3} \mathrm{He}$ wave functions, the purely isovector PS, $\mathrm{V}$ and $\pi \pi_{S}$ currents give non vanishing contributions to the $F_{M}^{S}\left(q_{\mu}\right)$ combination. However as the $\pi \pi_{S}$ contribution is very small, is not shown.

FIG. 4. Individual contributions to the $F_{M}^{V}\left(q_{\mu}\right)$ combination, Eq. (2.30), of the ${ }^{3} \mathrm{H}$ and ${ }^{3} \mathrm{He}$ magnetic form factors, obtained with the dipole parametrization of the nucleon electromagnetic form factors. The sign of each contribution is given in parenthesis. Note that, because of isospin-symmetry breaking components present in the ${ }^{3} \mathrm{H}$ and ${ }^{3} \mathrm{He}$ wave functions, the purely isoscalar $\rho \pi \gamma$ current gives non vanishing contributions to the $F_{M}^{V}\left(q_{\mu}\right)$ combination. However, being very small, it is not shown.

FIG. 5. The $F_{M}^{S}\left(q_{\mu}\right)$ combinations of the ${ }^{3} \mathrm{H}$ and ${ }^{3} \mathrm{He}$ magnetic form factors, obtained with single-nucleon currents $(1-\mathrm{N})$, and with inclusion of two-nucleon current $[(1+2)-\mathrm{N}]$ and $\pi \pi_{S}$ three-nucleon current (TOT-N) contributions, are compared with data (shaded area) from Amroun et al. [41. The dipole parametrization is used for the nucleon electromagnetic form factors.

FIG. 6. Same as in Fig. 5, but for the $F_{M}^{V}\left(q_{\mu}\right)$ combination of the ${ }^{3} \mathrm{H}$ and ${ }^{3} \mathrm{He}$ magnetic form factors.

FIG. 7. The charge form factors of ${ }^{3} \mathrm{H}$, obtained with a single-nucleon charge operator (1-N) and with inclusion of two-nucleon charge operator contributions (TOT-N), are compared with data (shaded area) from Amroun et al. [41]. Note that the 1-N results also include the Darwin-Foldy and spin-orbit corrections. Theoretical results correspond to the AV18/UIX PHH wave functions, and employ the dipole parametrization of the nucleon electromagnetic form factors. 
FIG. 8. Same as in Fig. 8, but for ${ }^{3} \mathrm{He}$.

FIG. 9. Individual contributions to the $F_{C}^{S}\left(q_{\mu}\right)$ combination, Eq. 2.31), of the ${ }^{3} \mathrm{H}$ and ${ }^{3} \mathrm{He}$ charge form factors, obtained with the dipole parametrization of the nucleon electromagnetic form factors. The sign of each combination is given in parenthesis. Note that, because of isospin-symmetry breaking components present in the ${ }^{3} \mathrm{H}$ and ${ }^{3} \mathrm{He}$ wave functions, the purely isovector $\omega \pi \gamma$ charge operator gives a non vanishing contribution to the $F_{C}^{S}\left(q_{\mu}\right)$ combination.

FIG. 10. Individual contributions to the $F_{C}^{V}\left(q_{\mu}\right)$ combination, Eq. (2.31), of the ${ }^{3} \mathrm{H}$ and ${ }^{3} \mathrm{He}$ charge form factors, obtained with the dipole parametrization of the nucleon electromagnetic form factors. The sign of each combination is given in parenthesis. Note that, because of isospin-symmetry breaking components present in the ${ }^{3} \mathrm{H}$ and ${ }^{3} \mathrm{He}$ wave functions, the purely isoscalar $\rho \pi \gamma$ charge operator gives a non vanishing contribution to the $F_{C}^{V}\left(q_{\mu}\right)$ combination.

FIG. 11. Transition correlation functions $u^{\sigma \tau I I}(r), u^{t \tau I I}(r)$, etc. obtained for the AV28Q model [50], and perturbation theory equivalents $u^{\sigma \tau I I, \mathrm{PT}}(r), u^{t \tau I I, \mathrm{PT}}(r)$, etc.

FIG. 12. $N \Delta$-transition two-body currents due to pion exchange.

FIG. 13. Diagrammatic representation of operators included in $j(\Delta)$ due to one-body currents $j^{(1)}(N \rightarrow \Delta), j^{(1)}(\Delta \rightarrow N)$ and $j^{(1)}(\Delta \rightarrow \Delta)$, and transition correlations $U^{N \Delta}, U^{\Delta N}, U^{\Delta \Delta}$, and corresponding hermitian conjugates. Wavy, thin, thick, dashed and cross-dashed lines denote photons, nucleons, $\Delta$-isobars and transition correlations $U^{B B^{\prime}}$ and $U^{B B^{\prime \dagger}}$, respectively.

FIG. 14. Diagrammatic representation of operators included in $j(\Delta)$ due to two-body currents $j^{(2)}(N N \rightarrow N \Delta), j^{(2)}(N N \rightarrow \Delta N)$, etc., and transition correlations $U^{N \Delta}, U^{\Delta N}$, and corresponding hermitian conjugates. Wavy, thin, thick, dashed and cross-dashed lines denote photons, nucleons, $\Delta$-isobars and transition correlations $U^{B B^{\prime}}$ and $U^{B B^{\prime \dagger}}$, respectively.

FIG. 15. Diagrams associated with connected three-body terms, which are neglected in the present work. Wavy, thin, thick, dashed, cross-dashed and dotted lines denote photons, nucleons, $\Delta$-isobars, transition correlations $U^{B B^{\prime}}$ and $U^{B B^{\prime}}{ }^{\dagger}$, and the two-body current $j^{(2)}(N N \rightarrow N N)$, respectively.

FIG. 16. The magnetic form factors of ${ }^{3} \mathrm{H}$, obtained with single-nucleon currents $(1-\mathrm{N})$, and with inclusion of two- and three-nucleon current (TOT-N) and $\Delta[$ TOT- $(\mathrm{N}+\Delta)]$ contributions.

FIG. 17. Same as in Fig. 16, but for ${ }^{3} \mathrm{He}$. 
FIG. 18. The single-nucleon contribution to the $F_{M}^{S}\left(q_{\mu}\right)$ combination of the ${ }^{3} \mathrm{H}$ and ${ }^{3} \mathrm{He}$ magnetic form factors is compared with the 1- $\Delta$ and 2- $\Delta$ contributions, associated respectively with diagrams of Fig. 13 and 14.

FIG. 19. The single-nucleon and leading PS two-nucleon contributions to the $F_{M}^{V}\left(q_{\mu}\right)$ combination of the ${ }^{3} \mathrm{H}$ and ${ }^{3} \mathrm{He}$ magnetic form factors are compared with the $1-\Delta$ and $2-\Delta$ contributions, associated respectively with diagrams of Fig. 13 and 14. 


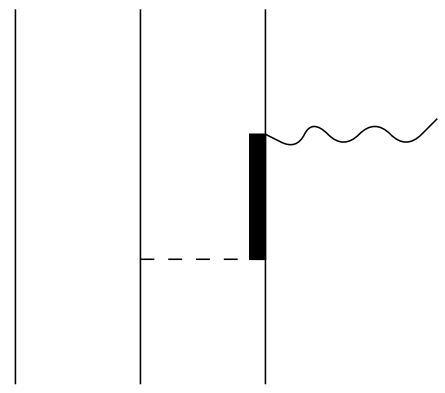

(a)

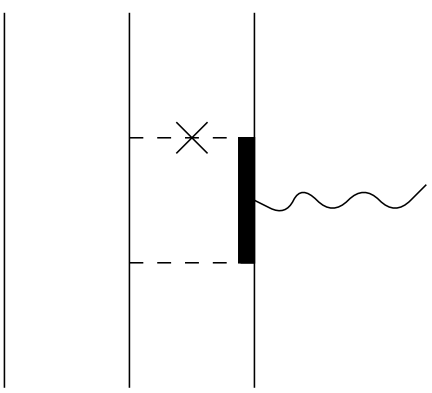

(e)

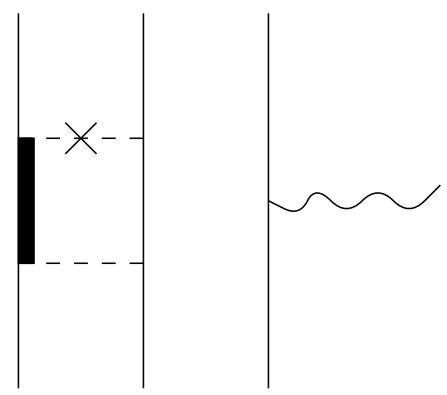

(h)

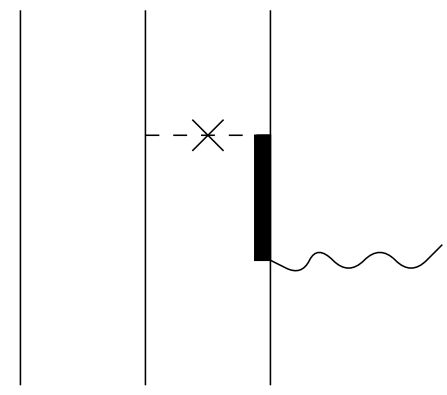

(b)

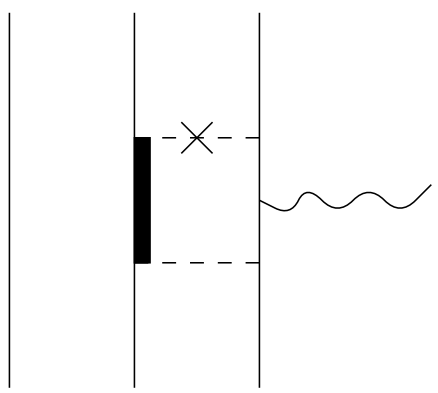

(f)

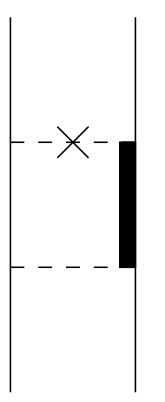

(i)

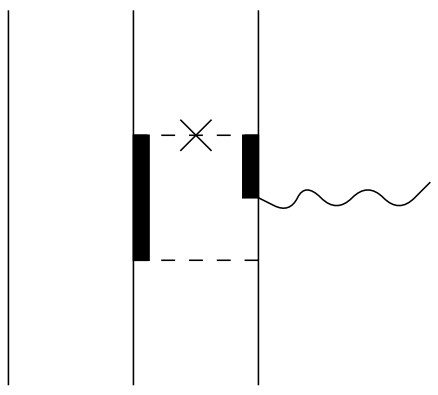

(c)

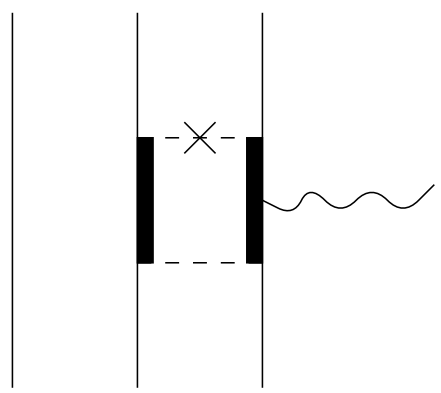

(g)

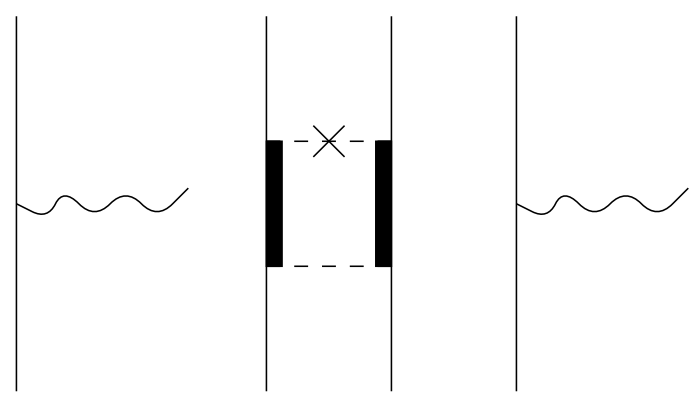

(j)

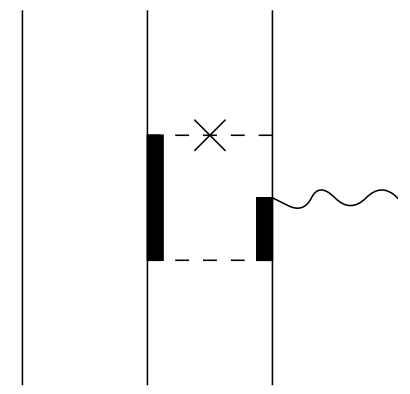

(d) 


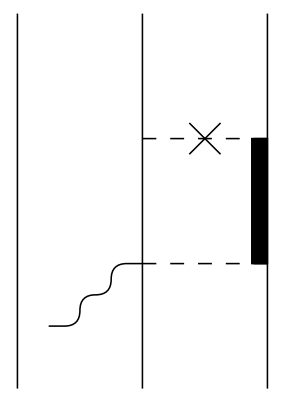

(a)

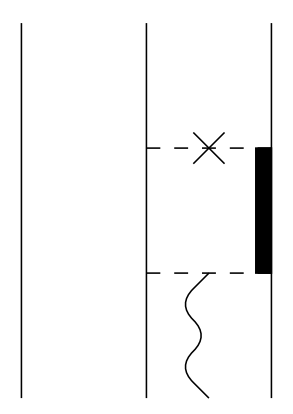

(e)

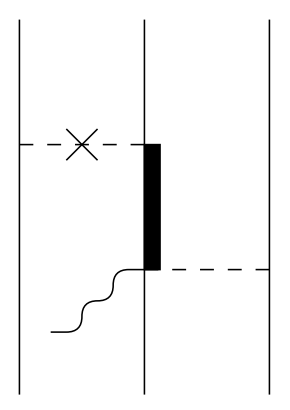

(i)

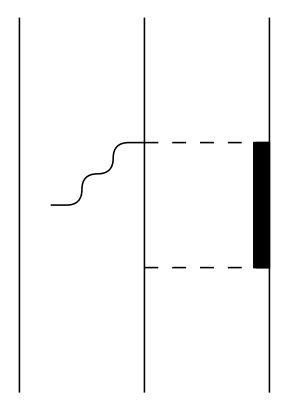

(b)

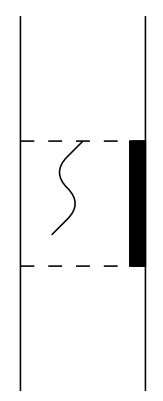

(f)

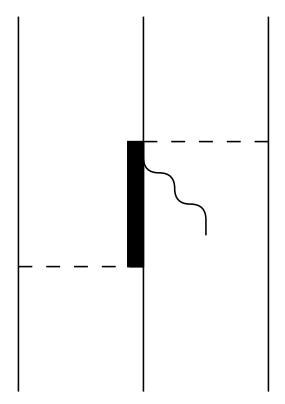

(j)

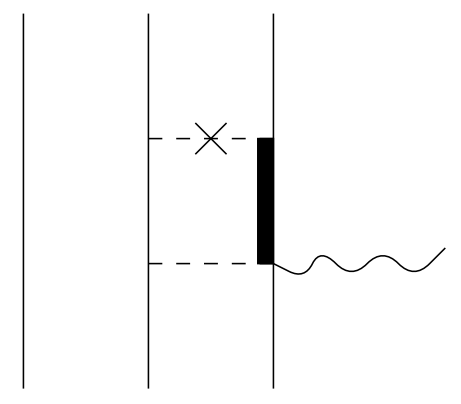

(c)

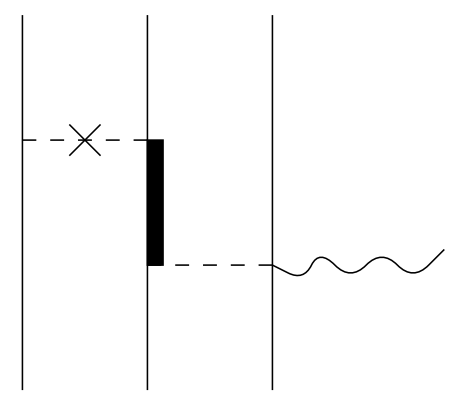

(g)

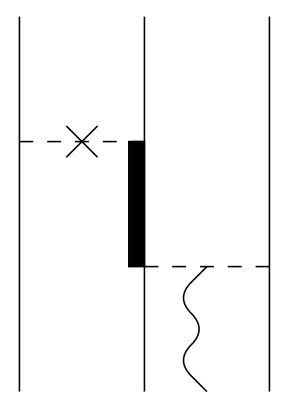

(k)

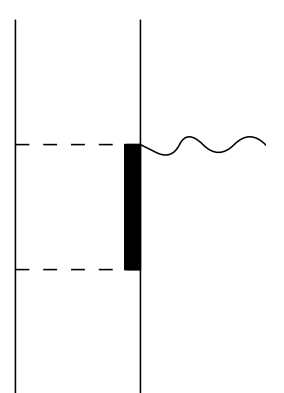

(d)

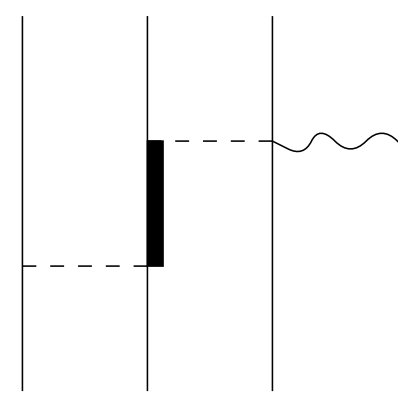

(h)

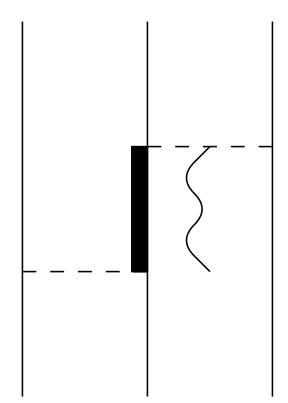

(1) 


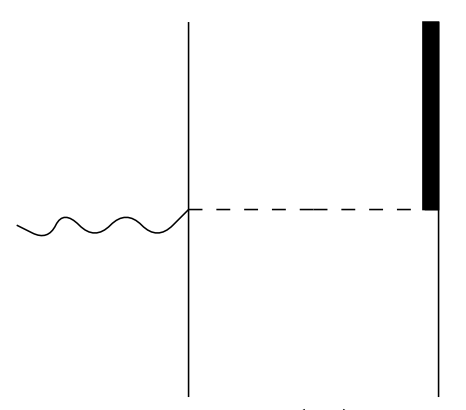

(a)



(b)

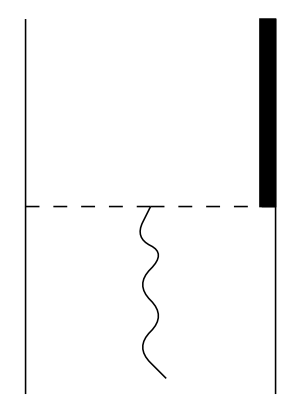

(c) 


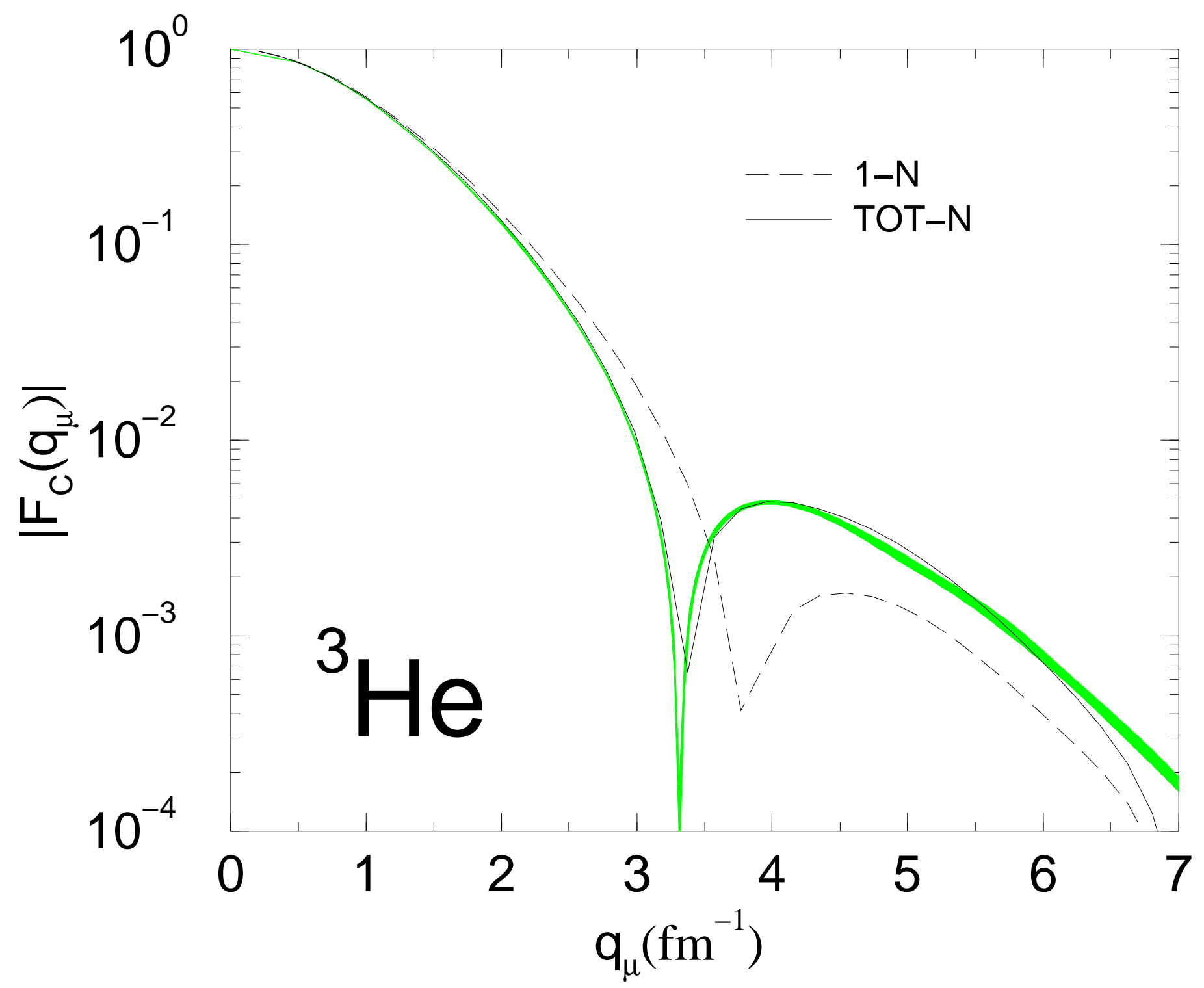




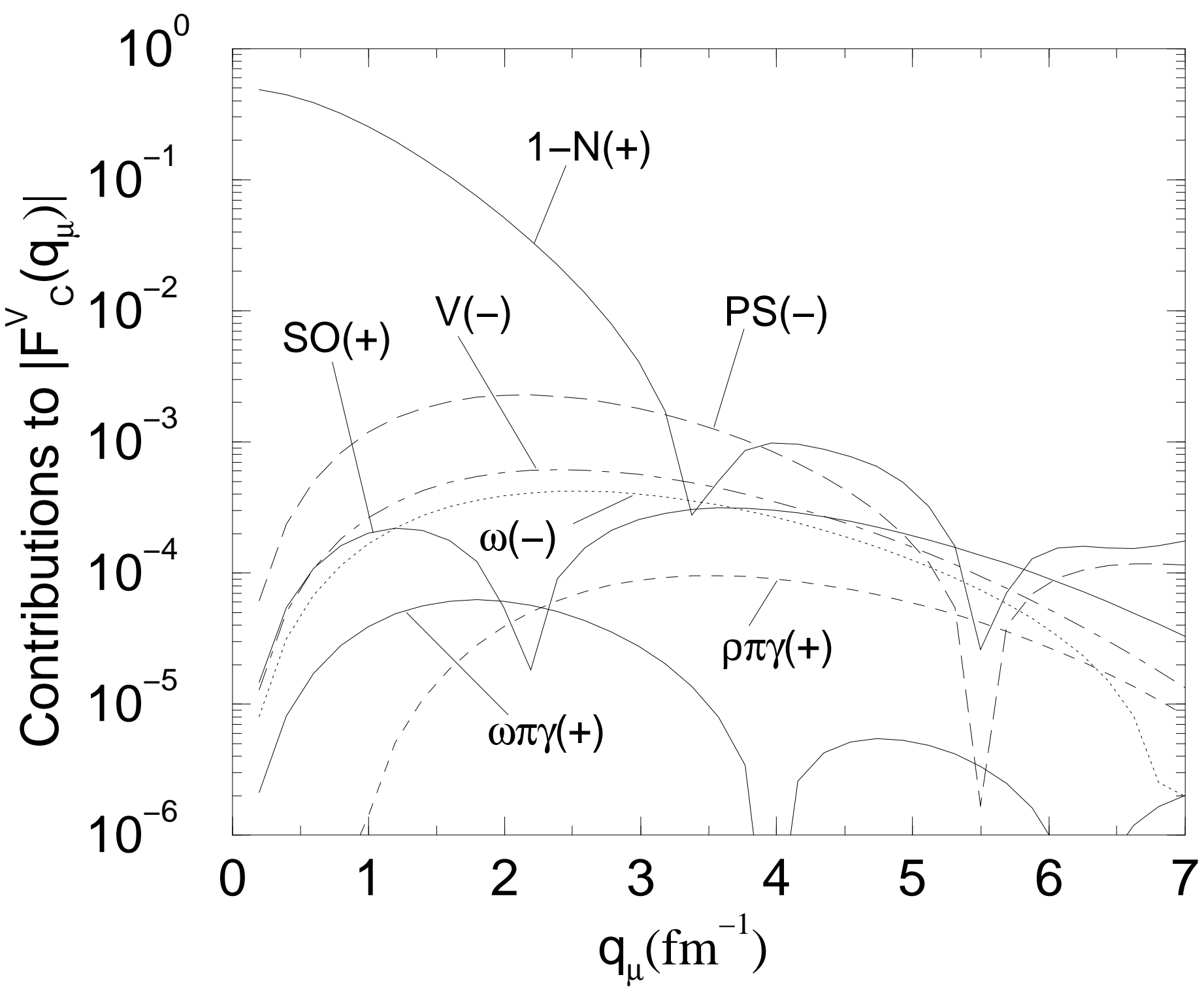




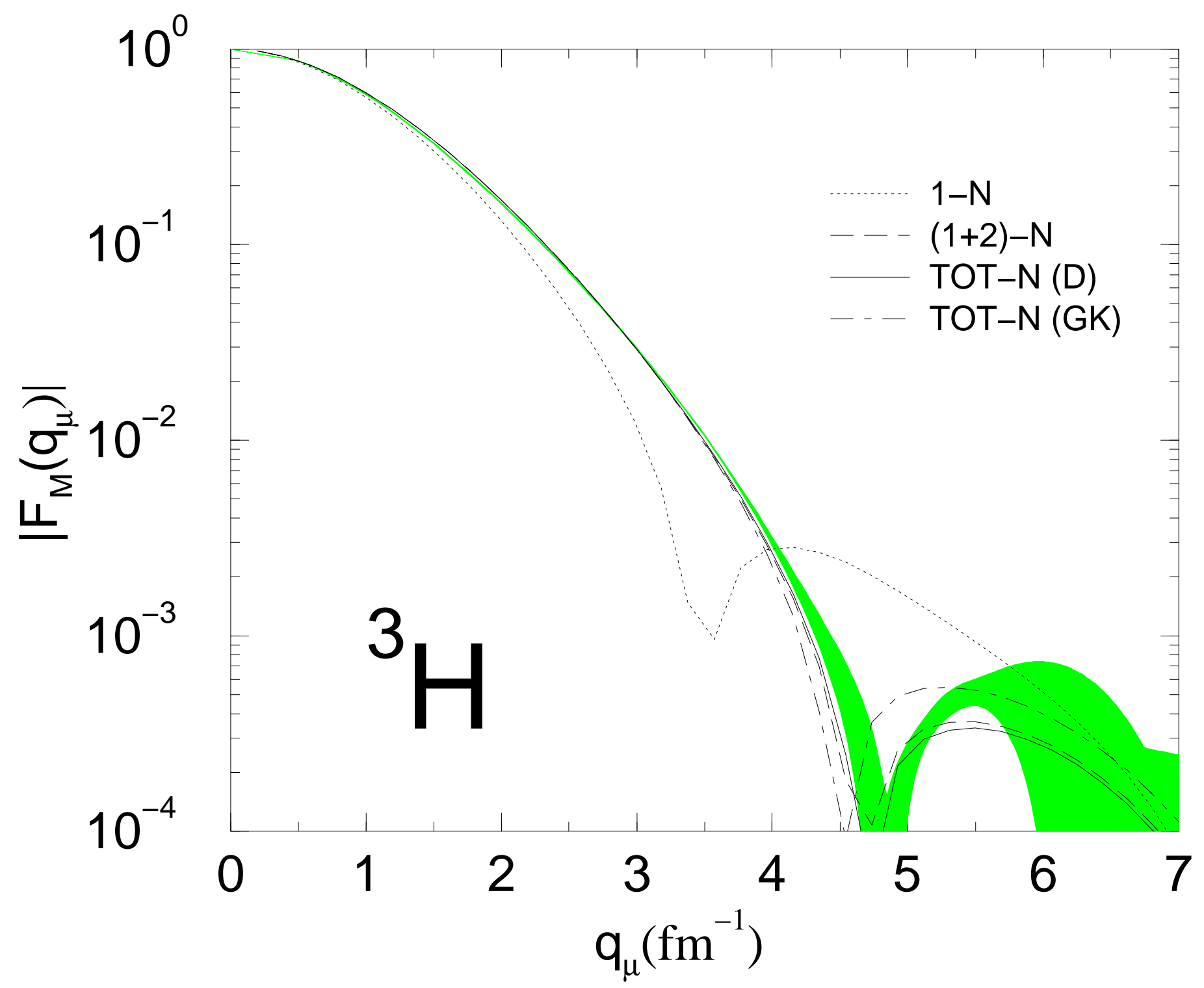




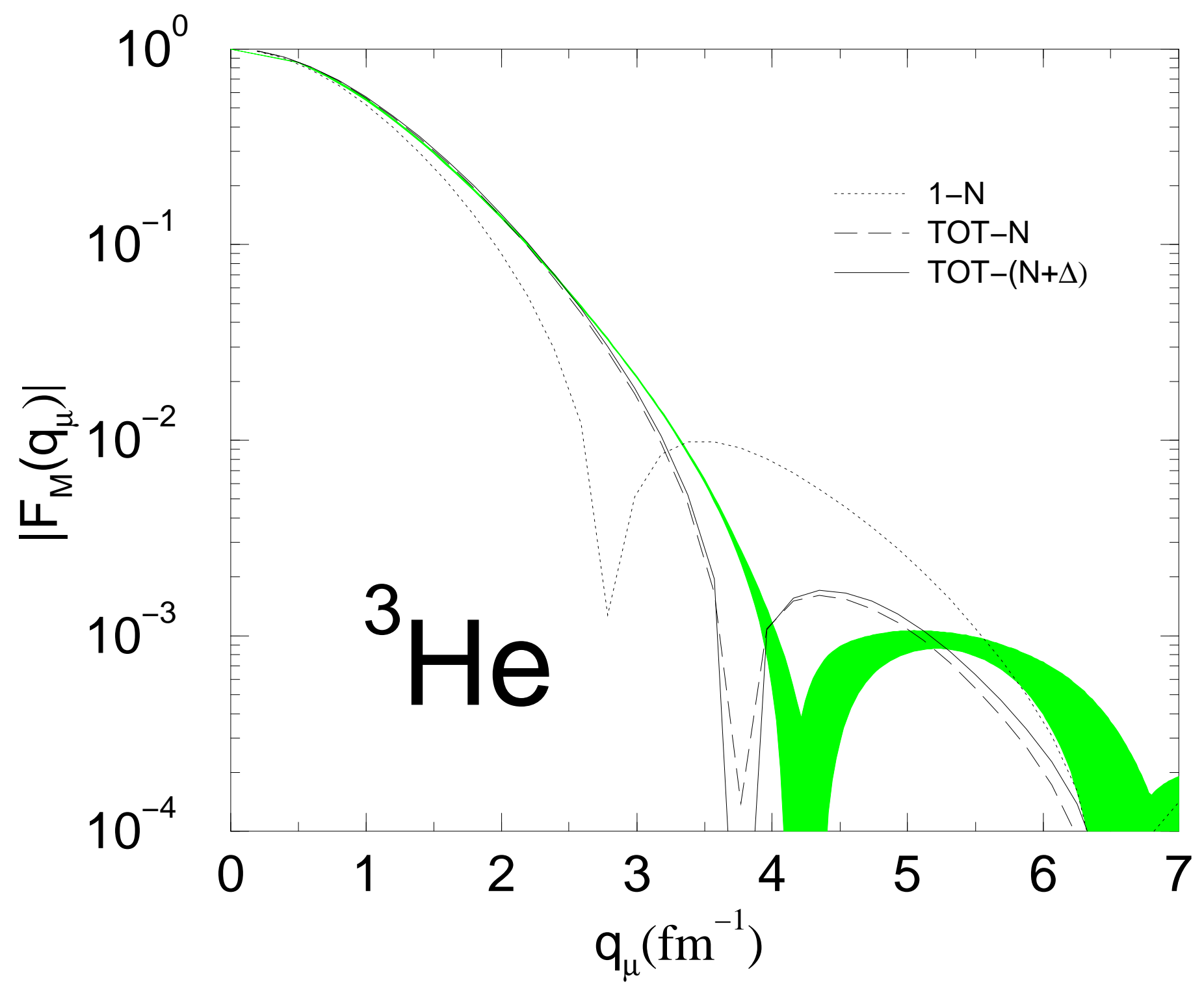




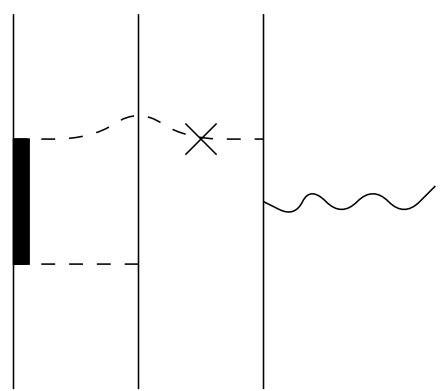

(a)

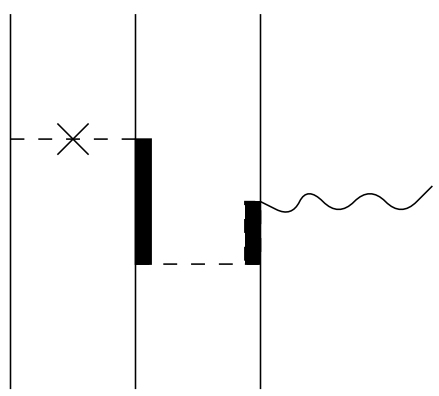

(b)

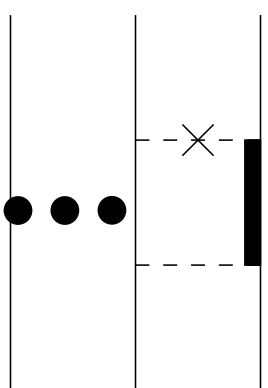

(c) 


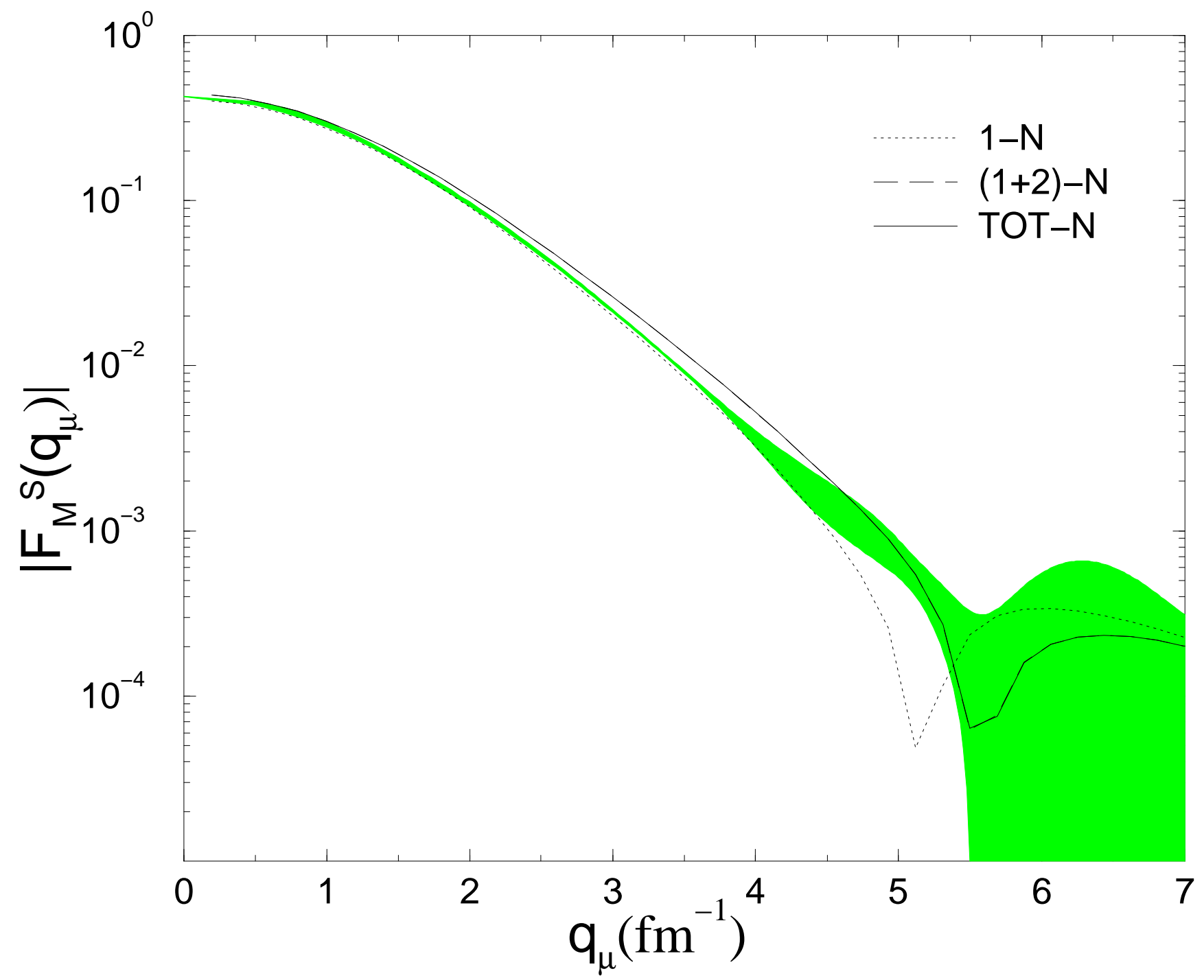




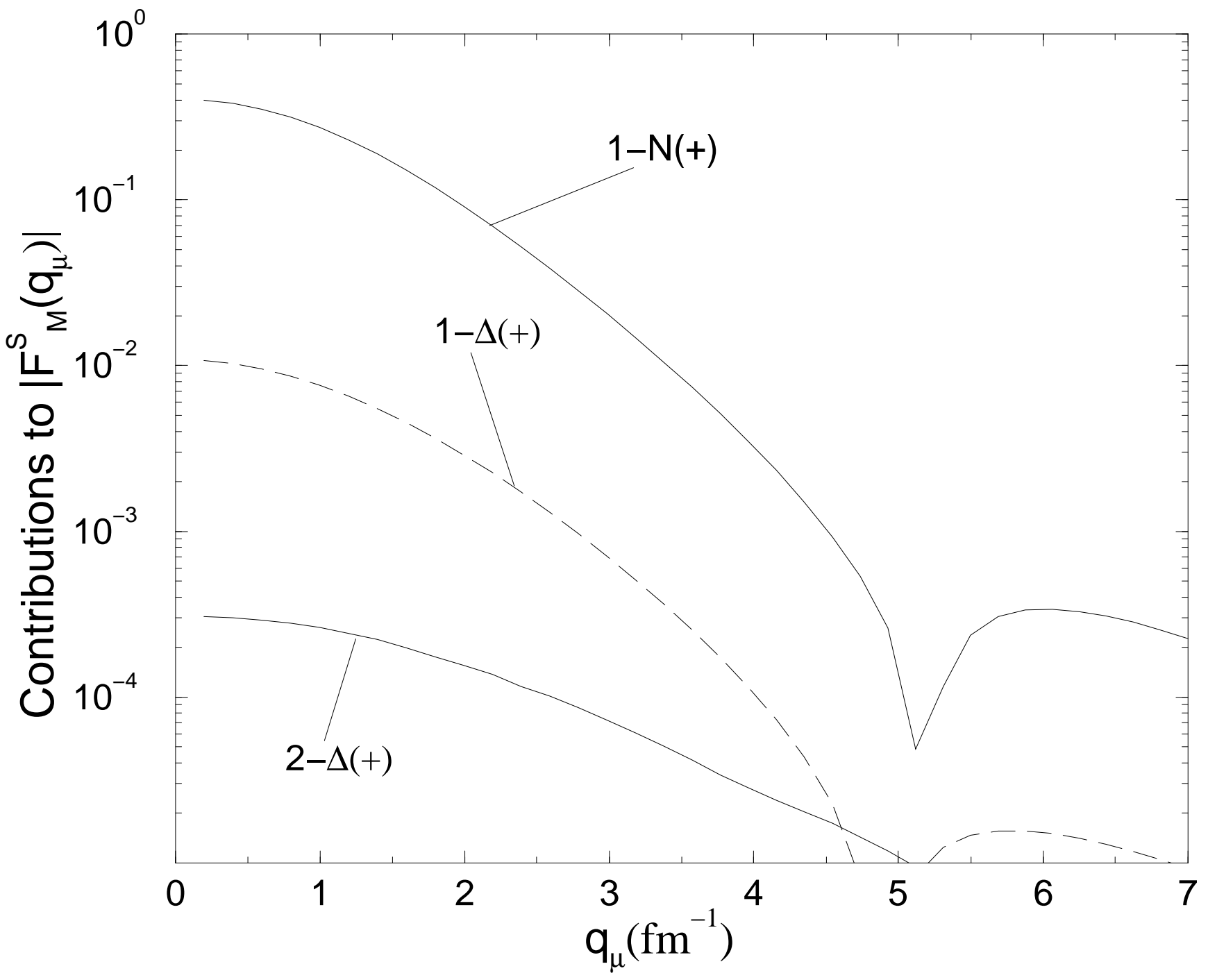




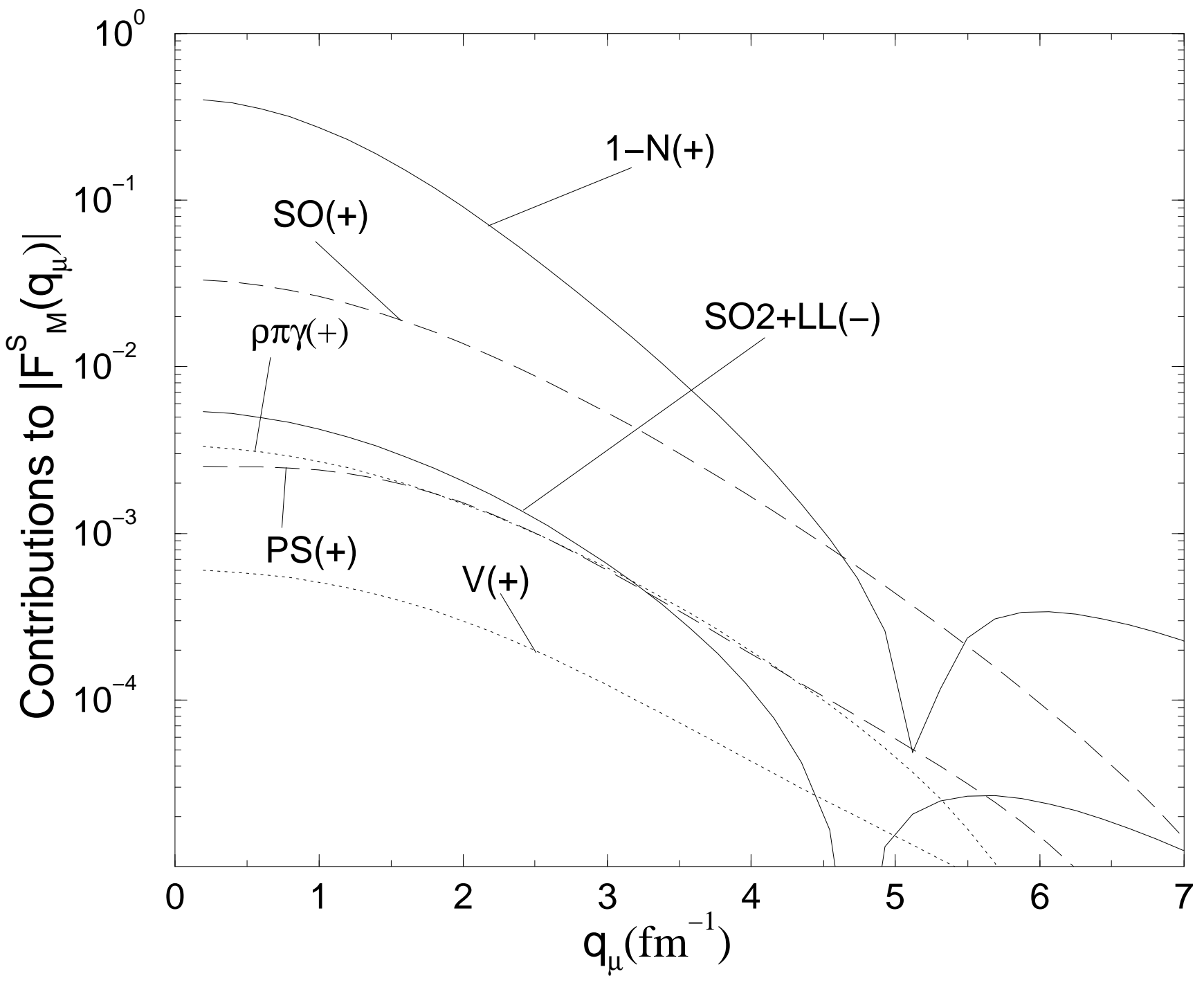




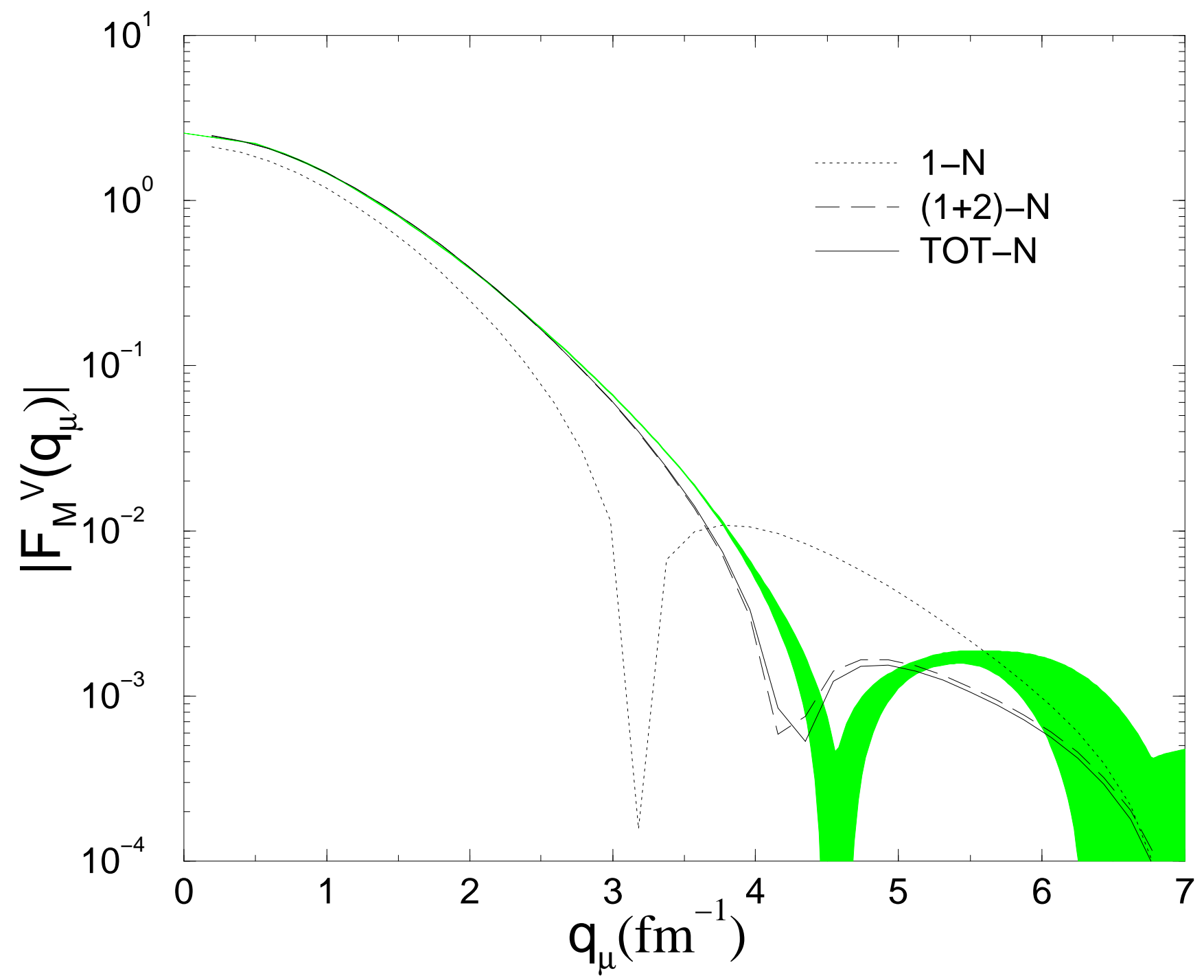




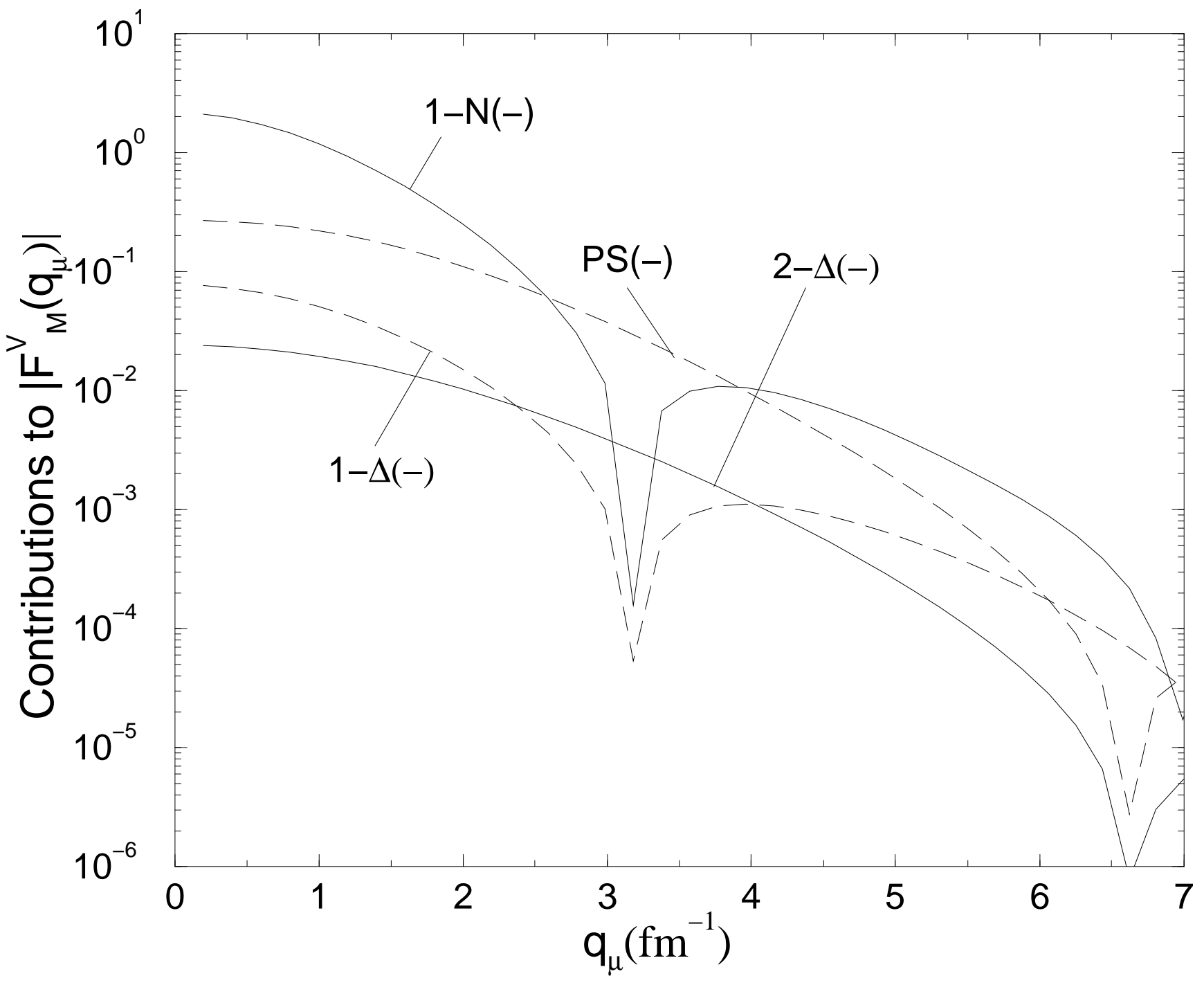




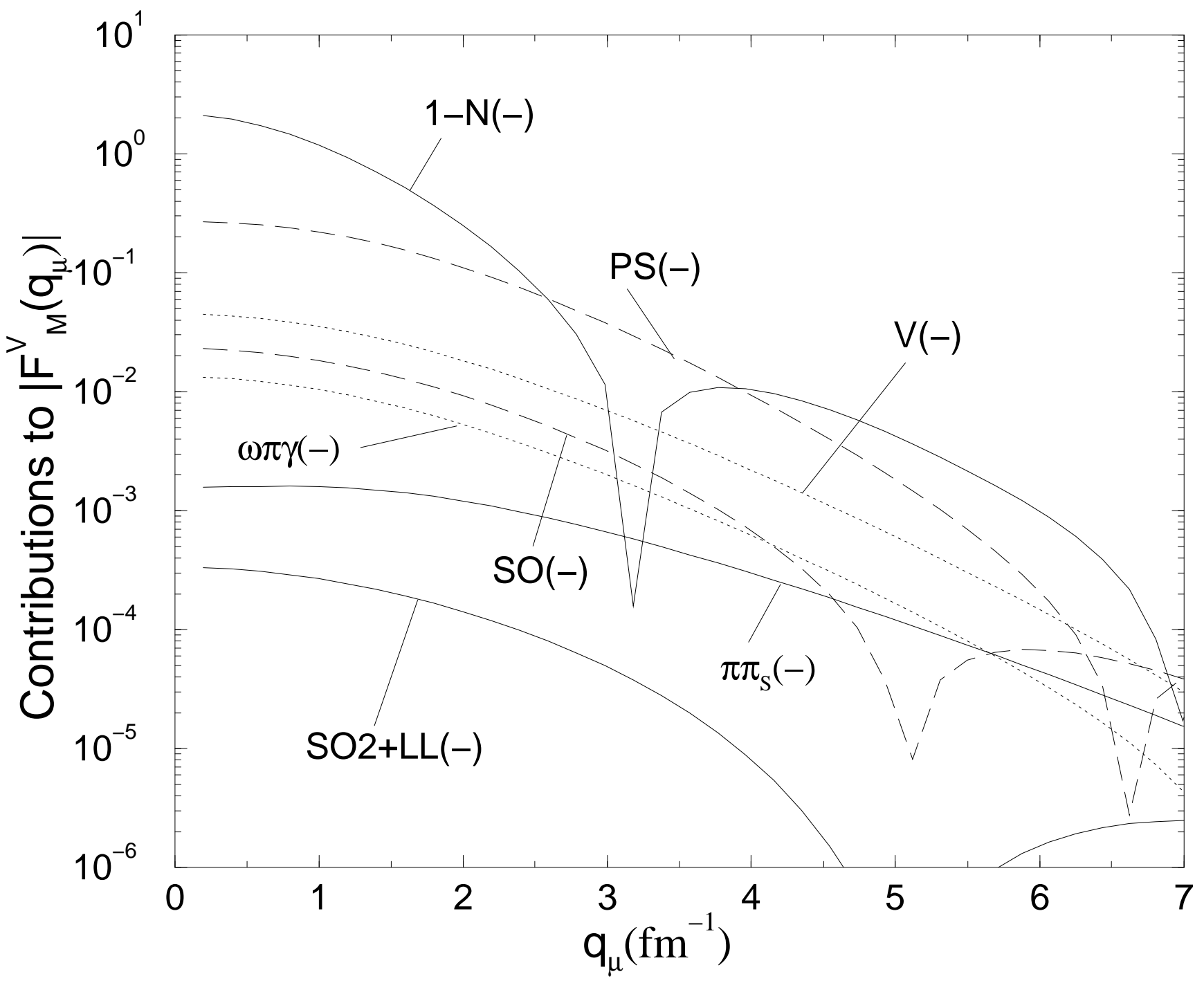




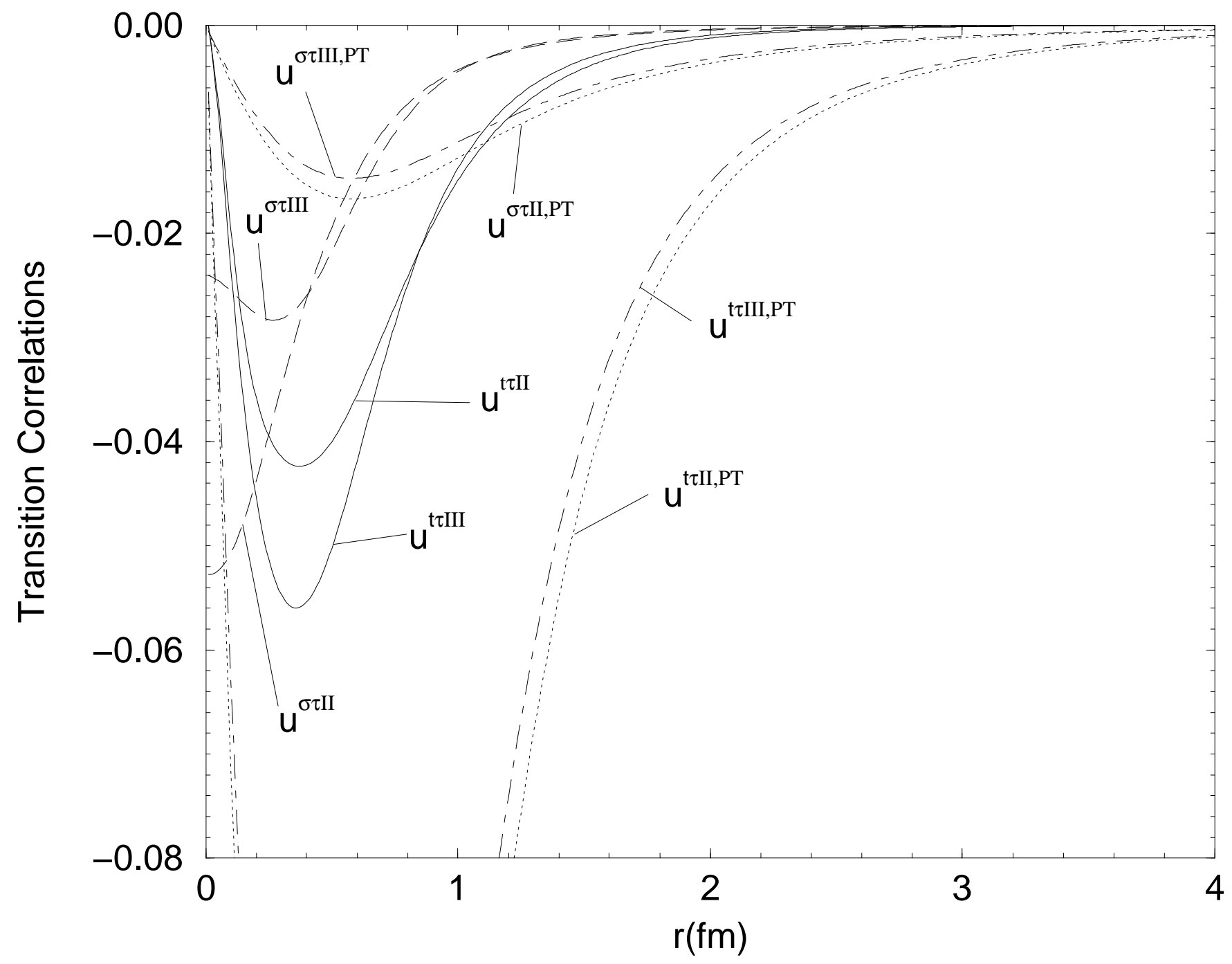

